TRANSACTIONS OF THE

AMERICAN MATHEMATICAL SOCIETY

Volume 350, Number 1, January 1998, Pages 213-250

S 0002-9947(98)01970-9

\title{
FILLING BY HOLOMORPHIC CURVES IN SYMPLECTIC 4-MANIFOLDS
}

\author{
RUGANG YE
}

\begin{abstract}
We develop a general framework for embedded (immersed) $J$ holomorphic curves and a systematic treatment of the theory of filling by holomorphic curves in 4-dimensional symplectic manifolds. In particular, a deformation theory and an intersection theory for $J$-holomorphic curves with boundary are developed. Bishop's local filling theorem is extended to almost complex manifolds. Existence and uniqueness of global fillings are given complete proofs. Then they are extended to the situation with nontrivial $J$ holomorphic spheres, culminating in the construction of singular fillings.
\end{abstract}

\section{INTRODUCTION}

In this paper we undertake a systematic study of filling by holomorphic curves (especially disks) in symplectic 4-manifolds. Here is an account of the main results.

A. Deformation of Embedded $J$-Holomorphic Curves. We develop a general framework for treating embedded (or immersed) $J$-holomorphic curves, with or without boundary. Embedded (or immersed) $J$-holomorphic curves can be treated in terms of $J$-holomorphic parametrizations. We present a more geometric approach, in which embedded (or immersed) $J$-holomorphic curves are considered as submanifolds. One advantage of this approach is that the Teichmüller spaces of conformal structures and automorphism groups can be avoided. The deformation theory becomes cleaner and more transparent.

Generic regularity. It is well-known that generic almost complex structures are "regular" or "Fredholm regular", meaning surjectivity of the linearized CauchyRiemann operator along simple closed $J$-holomorphic curves. This easily extends to curves with boundary on a fixed supporting surface. We prove that for each given almost complex structure, generic supporting surfaces are regular; see Theorem 3.3. This result is used in this paper for obtaining our general result on filling by holomorphic disks in the presence of nonconstant $J$-holomorphic spheres.

Regularity (genericity) under a Chern class (Maslov class) condition. In many situations, it suffices to use perturbation to generic parameters (almost complex structures and/or supporting surfaces). But in some important situations, such as filling by holomorphic disks, it is crucial to be able to show that a given parameter is regular or generic along a certain class of $J$-holomorphic curves. In [G], Gromov formulated a "genericity" result for closed embedded $J$-holomorphic curves

Received by the editors January 24, 1996.

1991 Mathematics Subject Classification. Primary 53C15; Secondary 32C25.

Partially supported by NSF. 
in 4 dimensions. Basically, genericity along a curve follows if the Chern class of the curve satisfies a natural inequality. We provide a proof of this result. Moreover, we extend the result to $J$-holomorphic curves with boundary: see Theorems 2.9 and 2.10. Our set-up is actually more general; namely, we handle a general linear Cauchy-Riemann operator on a complex line bundle over a Riemann surface. In the case of curves with boundary, the Chern class is replaced by the Maslov class. ${ }^{1}$

B. Positivity of Intersections and the Adjunction Formula. Two important tools in the theory of pseudo-holomorphic curves (in dimension 4) are positivity of intersection and the adjunction formula. In the case of closed curves, they were established by McDuff [M1] [M2] [M5], see also [MW]. Positivity of intersection for $J$-holomophic curves with boundary (in some crude formulation) has been known in the case when the almost complex structure $J$ is integrable and the supporting surface is real analytic. It was an open problem in the general situation.

Based on the asymptotic analysis in [Y1], we prove positivity of boundary intersections for $J$-holomorphic curves with boundary. Combined with McDuff's result, this yields positivity of intersection for $J$-holomorphic curves with boundary. We would like to emphasize that even the concept of intersection number for curves with boundary is nontrivial, and has not been well-understood before. We introduce a rigorous definition of it and show that it is a homotopy invariant.

In particular, this invariant is important for formulating the adjunction formula for $J$-holomorphic curves with boundary. (Unlike the adjunction formula for closed curves, where the classical situation is well-known, the case of curves with boundary does not seem to be a classical topic.) We establish the adjunction formula for $J$ holomorphic curves which are immersed along the boundary, which suffices for the applications in this paper. We plan to treat the general case in a subsequent paper.

The adjunction formula plays an important role in filling by holomorphic disks, especially in the presence of nonconstant $J$-holomorphic spheres.

C. Extension of Bishop's Theorem. Let $M$ be a 4-dimensional almost complex manifold with almost complex structure $J$, and $S$ a 2-dimensional submanifold in $M$. Let $p_{0} \in \stackrel{\circ}{S}$ be an elliptic complex point. We have the following:

Theorem 1. There is a unique (up to reparametrizations in $t$ ) smooth 1-parameter family of mutually disjoint, embedded J-holomorphic disks $\left\{F_{t}\right\}_{0<t<1}$ in $M$ such that $\partial F_{t} \subset S \backslash\left\{p_{0}\right\}$ for all $t$ and $F_{t}$ converges to $p_{0}$ as $t \rightarrow 0$. The family $\left\{F_{t}\right\}_{0<t<1}$ yields a smooth embedding of $B \times(0,1)$, where $B$ denotes the unit disk in $\mathbb{R}^{2}$. Moreover, $N=\left(\bigcup_{t} F_{t}\right) \cup\left\{p_{0}\right\}$ is a $C^{1}$ embedded J-flat half 3-ball (it is smooth away from $p_{0}$ ).

This is a generalization of the fundamental result of E. Bishop in [B] for complex manifolds. The higher dimensional version will appear elsewhere. We note that extension of Bishop's result to almost complex manifolds has resisted resolution for quite some time. The proof of Theorem 1 goes along lines which are rather different from the arguments in $[\mathrm{B}]$. The existence part is obtained by deforming suitable nearly $J$-holomorphic disks. The subtlety here is the degeneracy in the deformation set-up. The key for overcoming this difficulty is to balance the deformations in different directions carefully. On the other hand, the uniqueness follows from

\footnotetext{
${ }^{1}$ Added in proof: Hofer-Sikarov-Lizan and Lorek independently obtained similar results by different methods. Their preprints appeared after the present paper was circulated.
} 
stability of intersections, which is a consequence of positivity of intersection and the homotopy invariance of intersection.

D. Global Filling. The family $\left\{F_{t}\right\}$ is a local filling of $S$ by $J$-holomorphic disks. One attempts to extend it to a global filling under suitable assumptions on $S$ and $M$. We consider the case that $J$ is tamed by a symplectic form $\omega$, i.e. the quadratic form $\omega(\cdot, J)$ is positive definite everywhere. Moreover, we assume that $(M, \omega, J)$ has bounded geometry, i.e., $(M, g)$ has uniformly bounded sectional curvatures and positive injectivity radius, and $J$ has uniformly bounded covariant derivative, where $g$ is the Riemannian metric obtained from symmetrizing $\omega(\cdot, J)$. (The bounded geometry condition automatically holds if $M$ is compact.) We call such a $J$ uniformly tamed (by $\omega$ ). We shall first prove

Theorem 2. Assume that $J$ is uniformly tamed, $M$ has a $J$-convex boundary, $S$ is diffeomorphic to the 2-sphere $\mathbb{S}^{2}, S \subset \partial M$, and $S$ has exactly 2 complex points $p_{1}$ and $p_{2}$ which are elliptic. If $M$ contains no non-constant $J$-holomorphic sphere, then there exists a unique (up to reparametrizations in t) smooth family of mutually disjoint, embedded $J$-holomorphic disks $\left\{F_{t}\right\}_{0<t<1}$ in $M$ such that $\partial F_{t} \subset S \backslash\left\{p_{1}, p_{2}\right\}$ for all $t, \lim _{t \rightarrow 0} F_{t}=p_{1}$ and $\lim _{t \rightarrow 1} F_{t}=p_{2}$. The family $\left\{F_{t}\right\}_{0<t<1}$ yields a smooth embedding of $B \times(0,1)$. Moreover, $N=\left(\bigcup F_{t}\right) \cup\left\{p_{1}, p_{2}\right\}$ is a $C^{1}$ embedded $J$-flat 3-ball with $S$ as boundary (it is smooth away from $p_{1}$ and $p_{2}$ ).

For convenience, we shall henceforth call a surface $S \subset M$ simple, provided that it is diffeomorphic to $\mathbb{S}^{2}$ and has exactly two complex points which are elliptic. Theorem 2 was stated by M. Gromov in $[G]$ with a sketch of some ideas for proof. We point out that our proof can be extended to cover the case that $\partial M$ is only weakly $J$-convex. The details will be presented elsewhere. Besides Theorem 1 , another fundamental ingredient in proving Theorem 2 (and the other results below) is Gromov's compactness theorem for pseudoholomorphic curves, which we proved fully in [Y1]. Other tools are positivity and stability of intersection, and the adjunction formula mentioned before.

Note that the hypersurface $N$ in Theorem 2 or 1 is $J$-flat or Levi flat in the sense that it has vanishing Levi form; see Section 5 . If $M$ is Kähler, we can define the complex mean curvature of $N$ at a point $p \in N$ to be the trace of the second fundamental form of $N$ on the complex plane contained in $T_{p} N$. Then Levi-flatness means that the complex mean curvature of $N$ vanishes everywhere. Hence Levi flat surfaces can be compared with minimal surfaces. The condition of Levi flatness gives rise to a quasilinear degenerate elliptic system. Analytically, this system is more subtle than the classical minimal surface system.

A useful consequence of the proof of Theorem 2 is the following filling result (such a consequence was first observed by Eliashberg [E2]):

Theorem 3. Assume that $J$ is unformly tamed, and $M$ has a $J$-convex boundary and contains no nonconstant $J$-holomorphic sphere. Let $f$ be a J-holomorphic curve with boundary such that the image of its boundary is an embedded curve $L$ in $\partial M$. We further assume that $L$ bounds an embedded disk $S$ in $M$ which has exactly one complex point $p_{0}$, which is elliptic. Then $f$ is a (possibly branched) covering onto an embedded disk. If $f$ is an immersion, then it is actually an embedding. Moreover, there is a unique smooth 1-parameter family of mutually disjoint, embedded $J$ holomorphic disks $\left\{F_{t}\right\}_{0<t \leq 1}$ such that $\partial F_{t} \subset S \backslash\left\{p_{0}\right\}$ for all $t, F_{t}$ converges to $p_{0}$ as $t \rightarrow 0$, and $F_{1}$ is the image of $f$. The family $\left\{F_{t}\right\}$ along with the limit point $p_{0}$ 
yields a $C^{1}$ embedding of the (upper) half unit ball in $\mathbb{R}^{3}$. In particular, image $(f)$ is embedded and unknotted.

We would also like to mention the following basic application of filling by holomorphic disks due to Gromov: symplectically fillable 3-dimensional contact manifolds are tight. The proof of Theorem 2 can be applied directly to yield this result. Moreover, there seems to be hope to extend it to weak contact structures (confoliations in the terminology of Eliashberg). This will be pursued in the future.

E. Global Filling in the Presence of Nonconstant J-Holomorphic Spheres. The assumption in Theorem 2 (and Theorem 3) about $J$-holomorphic spheres is rather restrictive, because non-constant $J$-holomorphic spheres are often present. Non-constant $J$-holomorphic spheres constitute a main class of obstructions to global filling. It is important to understand these obstructions and obtain global filling in the presence of non-constant $J$-holomorphic spheres. There are three main problems here:

Problem 1. Characterize the obstructions.

Problem 2. Can one extend the local fillings (provided by Theorem 1) beyond obstructions to obtain global fillings with singularities?

Problem 3. Can one use suitable perturbation procedures to kill obstructions?

We solve all the three problems for rationally regular J. First, we introduce some terminology.

Definition 1. An "exceptional $J$-holomorphic sphere" in $M$ is an embedded $J$ holomorphic sphere $F$ with Chern class $c(F)=1$. An "exceptional $J$-cusp-curve" for a given surface $S$ is a $J$-cusp-curve $C$ consisting of an embedded $J$-holomorphic disk $F_{0}$ with boundary on $S^{*}=S \backslash\{$ complex points of $S\}$ and exceptional $J$ holomorphic spheres $F_{1}, \ldots, F_{k}$ (at least one) with the following properties. First, these spheres are mutually disjoint and disjoint from $S$. Second, each $F_{i}, i \geq 1$, intersects $F_{0}$ at a single point transversally. Third, the Maslov class $\mu(C)=2$, i.e. $\mu\left(F_{0}\right)=2-2 k$.

Definition 2. Let a symplectic form $\omega$ be given. An "exceptional symplectic sphere" is a symplectically embedded 2-sphere $F$ with self-intersection number $F \cdot F=-1$. An "exceptional symplectic cusp-curve" $C$ for a given surface $S \subset \partial M$ is the union of a symplectically embedded disk $F_{0}$ with $\partial F_{0} \subset S, T_{p} F_{0} \not \subset T_{p} \partial M$ for all $p \in \partial F_{0}$ and exceptional symplectic spheres $F_{1} \ldots, F_{k}$ (at least one) with the following properties. First, these spheres are mutually disjoint and disjoint from $\partial M$. Second, each $F_{i}, i \geq 1$, intersects $F_{0}$ at a single point transversally. Third, the self-intersection number $C \bullet C$ is equal to 0 , i.e. $F_{0} \bullet F_{0}=-2 k$. (For the definition of $\bullet$ we refer to Section 7.)

It turns out that exceptional $J$-cusp-curves are the only obstructions to smooth global filling in the case that $J$ is rationally regular. Indeed we have the following result for smooth global filling in the presence of non-constant $J$-holomorphic spheres:

Theorem 4. Assume that $J$ is unformly tamed and rationally regular, and $M$ has a J-convex boundary. Then the conclusion of Theorem 2 holds for every simple $S \subset \partial M$ for which no exceptional J-cusp-curve exists. On the other hand, given $S$, 
if an exceptional J-cusp-curve for $S$ exists, then there is no smooth global filling of $S$. Consequently, the conclusion of Theorem 2 holds for every simple $S \subset \partial M$ for which no exceptional symplectic cusp-curve exists.

A corresponding version of Theorem 3 holds; we omit the statement. Rational regularity is a generic property, i.e. the set of rationally regular (and uniformly tamed) $J$ is a countable intersection of open and dense sets. Here one can restrict to the space of $J$ which coincide with a given $J_{0}$ along $\partial M$.

As a corollary of Theorem 4 we obtain

Theorem 5. Assume that $(M, \omega)$ is minimal, i.e. it contains no exceptional symplectic sphere, $J$ is rationally regular and uniformly tamed by $\omega$, and $M$ has a $J$-convex boundary. Then the conclusion of Theorem 2 holds for every simple $S \subset \partial M$.

A corresponding version of Theorem 3 holds. We remark that, in general, minimal manifolds may contain nonconstant $J$-holomorphic spheres. Hence Theorem 5 does not follow from Theorem 2. On the other hand, consider $(M, \omega, J)$, where $J$ is uniformly tamed by $\omega$ and $M$ has a $J$-convex boundary. By the results in [M3], one can blow down a maximal collection of disjoint exceptional symplectic spheres to obtain a minimal $\left(M_{1}, \omega_{1}, J_{1}\right)$ such that $J_{1}$ coincides with $J$ along $\partial M_{1}=\partial M$. This combined with Theorem 5 gives strong information on the structure of $(M, \omega, J)$; see [E1]. For example, one obtains the following result of Eliashberg:

Corollary. Assume that $J$ is tamed by $\omega$, and $\partial M$ is $J$-convex and diffeomorphic to the 3-sphere $\mathbb{S}^{3}$. Then $M$ is diffeomorphic to the connected sum of the 4-ball $\mathbb{B}^{4}$ and several copies of $\mathbb{C} P^{2}$ with the negative orientation. If $(M, \omega)$ is minimal, then $M$ is diffeomorphic to $\mathbb{B}^{4}$.

By a recent result of Eliashberg, $(M, \omega)$ is actually, symplectomorphic to a pseudoconvex domain in $\mathbb{C}^{2}$ with a few points blown up. Another corollary of Theorem 4 is the following result (a corresponding version of Theorem 3 holds):

Theorem 6. Assume that $J$ is uniformly tamed and rationally regular, and $M$ has a $J$-convex boundary. Let $S \subset \partial M$ be simple. If $J$ is rationally $S$-regular (either in the embedding set-up or in the parametric set-up), then the conclusion of Theorem 2 holds for $S$.

The case of the embedding set-up is more involved than that of the parametric set-up. It is needed e.g. for proving Theorem 7 below. For the meaning of "rationally $S$-regular" we refer to Section 3 . By generic regularity mentioned before, this theorem implies that for a fixed $S$, the obstruction of $J$-holomorphic spheres to smooth global filling disappears after a suitable perturbation of $J$. On the other hand, for a given $J$ and an isotopy class $\mathcal{S}$ of $S \subset \partial M$, there is a generic family of $S$ in $\mathcal{S}$ such that $J$ is rationally $S$-regular in the embedding set-up for every $S$ in this family. Thus, the obstruction of $J$-holomorphic spheres also disappears after a suitable perturbation of $S$ while $J$ is being kept fixed. But we emphasize that in general the obstruction of $J$-holomrophic spheres cannot be killed simutaneously for a family of $S$ by perturbing $J$. On the other hand, a family of disjoint $S$ may develop intersections after perturbations of $S$ for the purpose of killing the obstruction. The 4 -ball $\mathbb{B}^{4}$ with one point blown up is a simple example.

Finally, we have the following general result on singular fillings: 
Theorem 7. Assume that $J$ is uniformly tamed and rationally regular, and $M$ has a J-convex boundary. Let $S \subset \partial M$ be simple. Then there is a unique (up to reparametrizations) singular filling of $S$, which is smooth if and only if there is no exceptional $J$-cusp-curve for $S$. Consequently, there is a J-flat hypersurface $N$ with finitely many interior singularities whose boundary is $S$.

Indeed, all the previous theorems on global filling can be considered as consequences of this result. Roughly speaking, a singular filling is a global filling with finitely many singularities in the interior (the singular set may be empty). Singular fillings are significant from both the geometric and the analytic point of view. For example, the singular filling of $S$ captures all $J$-holomorphic curves with non-empty boundary on $S$; see Theorem 8.8 and the Remark to Proposition 5.6.

F. Singularity Structure. We have a rather complete understanding of the structure of the singularities in singular fillings. In particular, the blow-up limit at a singularity is unique; see Theorem 8.7. This implies in particular Hausdorff convergence of smooth leaves in a singular filling to singular leaves with blow-up limits attached, which are nonconstant $J$-holomorphic spheres. This is an important property.

From the point of view of comparison with minimal surfaces as mentioned before, we should like to point out that blow-up in singular fillings is analogous to blowup of minimal spheres as studied in [SU] as well as blow-up of $J$-holomorphic curves (see e.g. [Y1]). One can also compare with tangent cone blow-up of areaminimizing varieties and energy-minimizing maps; see [S1] [S2] and [S3]. A basic question in those theories is whether blow-up limits are unique. Allard-Almgren [AA] and Simon [S1] [S2] [S3] have obtained fundamental unique blow-up results for area-minimizing varieties and energy-minimizing maps. Their techniques are very profound. Besides these results, very few results on unique blow-up are known. Our unique blow-up result for singular fillings can be viewed from this perspective. The existence and uniqueness of singular fillings can also be viewed in this comparison.

In $[\mathrm{BK}]$, Bedford and Klingenberg obtained global filling in the presence of hyperbolic complex points. Their result is valid for Stein manifolds. With the framework developed in this paper at hand, it is reasonable to believe that their results should generalize to almost complex manifolds. The difficulty here is that the behavior of $(J$-)holomorphic disks at a hyperbolic complex point is very intricate. We shall treat this problem in a subsequent paper.

A general theory of filling by holomorphic disks has been formulated by Eliashberg in [E1]. Applications of filling by holomorphic disks are also given in [E1] (see also [E2]). In [H], Hofer presented an independent treatment of filling by holomorphic disks and an application to the Weinstein conjecture. Another independent treatment and an application to the camel problem were given by McDuff and Traynor in $[\mathrm{MT}]$. Earlier results on global filling were obtained e.g. by Bedford and Gaveau [BG]. Further references can be found in [E1].

We would like to mention that we obtained those parts of the proof of Theorem 2 other than Theorem 1 quite some time ago.

I am grateful to Y. Eliashberg for introducing me to the subject of filling by holomorphic curves. The writing of the previous version of this paper [Y2], which contains Theorems 1 and 2, was finished and also part of the remaining results were obtained at Centre de Mathématiques et de Leurs Applications, ENS Cachan and Université de Paris Sud. Part of the results were obtained at Forschungsinstitut für 
Mathematik, ETH. I thank all three institutions for their support and hospitality. Specifically, I thank Professors J. Moser and J. M. Coron for the kind invitations. I also acknowledge a visit to Newton Institute for Mathematical Sciences, Cambridge, during which the exposition in this paper was improved.

\section{Complex Tangency}

Consider $S \subset M$ as described at the beginning of the introduction. A complex point of $S$ is a point $p$ such that the tangent plane $T_{p} S$ is complex, i.e. $J\left(T_{p} S\right)=$ $T_{p} S$. If $T_{p} S \cap J\left(T_{p} S\right)=\{0\}$, then $p$ is called a real point. Note that points of $S$ are either complex or real.

Fix a complex point $p_{0} \in S$. We choose local coordinates around $p_{0}$ such that $p_{0}$ corresponds to the origin and $S$ (near $\left.p_{0}\right)$ is represented by the $\left(x_{1}, x_{2}\right)$-plane. Moreover, $J_{p_{0}}$ induces the standard almost complex structure $J_{0}$ on $\mathbb{R}^{4}$, i.e.

$$
\begin{gathered}
J_{p_{0}} \frac{\partial}{\partial x_{1}}=\frac{\partial}{\partial x_{2}}, \quad J_{p_{0}} \frac{\partial}{\partial x_{2}}=-\frac{\partial}{\partial x_{1}}, \\
J_{p_{0}} \frac{\partial}{\partial x_{3}}=\frac{\partial}{\partial x_{4}}, \quad J_{p_{0}} \frac{\partial}{\partial x_{4}}=-\frac{\partial}{\partial x_{3}} .
\end{gathered}
$$

Such coordinates will be called "preferred". It is convenient to work on the domain $U$ of the chosen coordinates, which is a neighborhood of the origin in $\mathbb{R}^{4}$. We still use the same letter $J$ to denote the induced almost complex structure on $U$. Consider the matrix funtion $\left(J_{i j}\right)$ defined as follows:

$$
J \frac{\partial}{\partial x_{i}}=\sum_{j=1}^{4} J_{i j} \frac{\partial}{\partial x_{j}}, \quad 1 \leq i \leq 4 .
$$

Along the $\left(x_{1}, x_{2}\right)$-plane we have

$$
\begin{aligned}
& J_{13}=a x_{1}+b x_{2}+O\left(x_{1}^{2}+x_{2}^{2}\right), \\
& J_{14}=c x_{1}+d x_{2}+O\left(x_{1}^{2}+x_{2}^{2}\right),
\end{aligned}
$$

for some constants $a, b, c$ and $d$.

It is easy to see that

$$
\begin{aligned}
& J_{23}=J_{14}+O\left(|x|^{2}\right), \\
& J_{24}=-J_{13}+O\left(|x|^{2}\right),
\end{aligned}
$$

where $x=\left(x_{1}, x_{2}, x_{3}, x_{4}\right)$. Hence the expansion (1.2) yields a similar expansion for $J_{23}$ and $J_{24}$ along the $\left(x_{1}, x_{2}\right)$-plane.

Definition 1.1.

$$
\begin{gathered}
\operatorname{ind}\left(p_{0}\right)=1 \text { if } a d-b c>0, \\
\operatorname{ind}\left(p_{0}\right)=-1 \text { if } a d-b c<0, \\
\operatorname{ind}\left(p_{0}\right)=0 \text { if } a d-b c=0 .
\end{gathered}
$$

Lemma 1.2. ind $\left(p_{0}\right)$ is an invariant, i.e. it is independent of the choice of preferred coordinates. 
Proof. This follows from an elementary computation. Set

$$
\mathbf{J}_{2}=\left(\begin{array}{ll}
J_{13} & J_{14} \\
J_{23} & J_{24}
\end{array}\right) \text {. }
$$

Consider new coordinates $x^{\prime}$ and let $A=\left(a_{i j}\right)$ be the Jacobian of the coordinate transformation, i.e.

$$
\frac{\partial}{\partial x_{i}^{\prime}}=\sum_{j=1}^{4} a_{i j} \frac{\partial}{\partial x_{j}}, \quad 1 \leq i \leq 4 .
$$

Then $A$ has the following form along the $\left(x_{1}, x_{2}\right)$-plane:

$$
A=\left(\begin{array}{ll}
A_{1} & 0 \\
A_{3} & A_{4}
\end{array}\right) \text {. }
$$

It follows that $\mathbf{J}_{2}^{\prime}=A_{1} \mathbf{J}_{2} A_{4}^{-1}$. On the other hand, since $J^{\prime}(0)=J_{0}$, we have

$$
A_{1}(0)=\left(\begin{array}{ll}
\alpha & -\beta \\
\beta & \alpha
\end{array}\right), \quad A_{4}(0)=\left(\begin{array}{ll}
\alpha_{1} & -\beta_{1} \\
\beta_{1} & \alpha_{1}
\end{array}\right) .
$$

for some $\alpha, \beta, \alpha_{1}, \beta_{1}$ with $\alpha^{2}+\beta^{2}>0$ and $\alpha_{1}^{2}+\beta_{1}^{2}>0$. Consequently, as far as the change of $\mathbf{J}_{2}$ along the $\left(x_{1}, x_{2}\right)$-plane is concerned, modulo higher order terms the coordinate transformation can be assumed to have the following form:

$$
\left\{\begin{array}{l}
x_{1}=\alpha x_{1}^{\prime}+\beta x_{2}^{\prime}, \\
x_{2}=-\beta x_{1}^{\prime}+\alpha x_{2}^{\prime}, \\
x_{3}=\alpha_{1} x_{3}^{\prime}+\beta_{1} x_{4}^{\prime}, \\
x_{4}=-\beta_{1} x_{3}^{\prime}+\alpha_{1} x_{4}^{\prime} .
\end{array}\right.
$$

We deduce (along the $\left(x_{1}, x_{2}\right)$-plane)

$$
\begin{aligned}
J_{13}^{\prime}= & \frac{1}{\alpha_{1}^{2}+\beta_{1}^{2}}\left\{\left[\left(\alpha \alpha_{1}-\beta \beta_{1}\right)(a \alpha-b \beta)-\left(\alpha \beta_{1}+\beta \alpha_{1}\right)(c \alpha-d \beta)\right] x_{1}^{\prime}\right. \\
& \left.+\left[\left(\alpha \alpha_{1}-\beta \beta_{1}\right)(a \beta+\alpha b)-\left(\alpha \beta_{1}+\beta \alpha_{1}\right)(c \beta+\alpha d)\right] x_{2}^{\prime}\right\} \\
& +O\left(\left(x_{1}^{\prime}\right)^{2}+\left(x_{2}^{\prime}\right)^{2}\right), \\
J_{14}^{\prime}= & \frac{1}{\alpha_{1}^{2}+\beta_{1}^{2}}\left\{\left[\left(\alpha \beta_{1}+\beta \alpha_{1}\right)(a \alpha-b \beta)+\left(\alpha \alpha_{1}-\beta \beta_{1}\right)(c \alpha-d \beta)\right] x_{1}^{\prime}\right. \\
& \left.+\left[\left(\alpha \beta_{1}+\beta \alpha_{1}\right)(a \beta+\alpha b)+\left(\alpha \alpha_{1}-\beta \beta_{1}\right)(c \beta+\alpha d)\right] x_{2}^{\prime}\right\} \\
& +O\left(\left(x_{1}^{\prime}\right)^{2}+\left(x_{2}^{\prime}\right)^{2}\right) .
\end{aligned}
$$

To simplify computations, we decompose the transformation into two steps: first assume $\alpha_{1}=1, \beta_{1}=0$; then assume $\alpha=1, \beta=0$. It is easy to check that $\operatorname{ind}\left(p_{0}\right)$ is invariant in both steps.

Remark. It is not hard to show that ind $\left(p_{0}\right)$ equals the intersection index of the Gauss map of $S$ with complex Grassmannians as discussed in [E1].

Definition 1.3. The complex point $p_{0}$ is called elliptic, hyperbolic or parabolic, if $\operatorname{ind}\left(p_{0}\right)=1,-1$ or 0 respectively. 
It is easy to see that this definition agrees with the classical definition for complex manifolds. By a transversality argument one can show that parabolic complex points do not occur generically. In other words, a suitable small perturbation of a given surface has no parabolic complex points.

Next we simplify the expansion (1.2) by suitable coordinate changes. First we achieve $a+d=0$. If this does not hold, we perform the transformation (1.4) with $\alpha=1, \beta=0, \alpha_{1}=\frac{c-b}{a+d}$ and $\beta_{1}=1$. In the new coordinates we then have $a+d=0$. Now assume $a+d=0$. If $a \neq 0$, we perform (1.4) with $\alpha_{1}=1, \beta_{1}=0, \beta=1$ and $\alpha$ being a solution of the equation $\alpha^{2}-\frac{b+c}{a} \alpha-1=0$. In the new coordinates we then have $a=d=0$. We arrive at

Lemma 1.4. We can choose preferred coordinates such that $a=d=0$.

This elementary lemma is important for constructing nearly $J$-holomorphic disks.

\section{Deformation of EMBEDDED $J$-HOLOMORPhiC CURVES}

A $C^{1}$ map $f$ from a Riemann surface $\Sigma$ into $M$ is called $J$-holomorphic if $J \circ d f=$ $d f \circ j$, where $j$ is the almost complex structure of $\Sigma$. A $J$-holomorphic $f$ is called a $J$-holomorphic curve. If $f$ is an immersion, we can consider the equivalence class of immersions $[f]$ containing $f$. (Two immersions are equivalent if they differ by a diffeomorphism of the domain.) We say that $[f]$ is an immersed submanifold.

Definition 2.1. $[f]$ is called $J$-holomorphic, provided that the image of $(d f)_{z}$ is a complex plane for every $z \in \Sigma$ and $f \in[f]$. If $f$ is an embedding, we identify $[f]$ with image $(f)$.

If $[f]$ is $J$-holomorphic, then there is a Riemann surface $\Sigma$ and a $J$-holomorphic map $\tilde{f}: \Sigma \rightarrow M$ such that $\tilde{f} \in[f]$. This follows from the integrability of almost complex structures in dimension 2.

We shall present a treatment of embedded $J$-holomorphic curves. With slight modifications, it also applies to immersed curves; we leave the details to the reader. To begin with, let us extend $J$ to 2-vectors: $J\left(v_{1} \wedge v_{2}\right)=J v_{1} \wedge J v_{2}$, and then extend it linearly. Let $g$ be a Riemannian metric on $M$ and $g_{J}$ its associated Hermitian metric: $2 g_{J}(\cdot, \cdot)=g(\cdot, \cdot)+g(J \cdot, J \cdot)$. We use $g_{J}$ in the following discussion. Consider an oriented embedded surface $F$. Locally on $F$, choose an oriented orthonormal tangent frame field $v_{1}, v_{2}$. The simple 2-vector field $v_{1} \wedge v_{2}$ is independent of the choice of the frame. Hence we obtain a global 2-vector field $w=v_{1} \wedge v_{2}$ on $F$. Indeed, at every $p \in F$, there is a unique oriented simple 2-vector of unit length which is tangent to $F$.

Lemma 2.2. $F$ is J-holomorphic if and only if $w-J(w)=0$.

Proof. If $F$ is $J$-holomorphic, then the equation is obvious if we choose $v_{2}=J v_{1}$. On the other hand, if $T_{p} F$ is not $J$-invariant at some point $p \in F$, then $v_{1}, v_{2}, J v_{1}$ and $J v_{2}$ consititute a base at $p$, whence $J v_{1} \wedge J v_{2}$ and $v_{1} \wedge v_{2}$ are two distinct members in a base for the space of 2-vectors at $p$.

We choose $H(F)=w-J(w)$ to be our nonlinear Cauchy-Riemann operator. (Of course, it depends on the choice of the metric $g$.) The 2-vector field $H(F)$ is anti- $J$ invariant, i.e. $J(H(F))=-H(F)$. Let $\Lambda(M)$ denote the bundle of anti- $J$-invariant 2 -vectors. Its fiber dimension is 2 . Indeed, let $v, J v, \tilde{v}$ and $J \tilde{v}$ be a base of $T_{p} M$ for some $p$. Then a base of $\Lambda_{p}(M)$ is given by $v \wedge \tilde{v}-J v \wedge J \tilde{v}$ and $v \wedge J \tilde{v}+J v \wedge \tilde{v}$. 
Lemma 2.3. Let $F$ be J-holomorphic and $V$ the normal bundle of $F$. Then there is a natural isomorphism $\Phi:\left.\Lambda(M)\right|_{F} \rightarrow \Lambda^{0,1}(V)$, where the last denotes the bundle of anti-complex linear maps from $\left(T_{p} F, J\right)$ to $\left(V_{p}, J\right), p \in F$.

Proof. Using duality we can define the interior product $\lfloor$ of a tangent vector to a 2 -vector. The desired isomorphism is then given by $\Phi(w)(v)=v\lfloor w$.

Now consider an embedded $J$-holomorphic curve $F$. We assume that either $F$ is closed or its boundary lies on $S^{*}=S \backslash\{$ complex points of $S\}$ for a compact surface $S$. In the following, we present a treatment of the latter case in detail. It applies directly to the former case: one just forgets the boundary and makes the related easy modifications. Choose a small positive number $\epsilon$ such that $\partial F \subset S_{\epsilon} \equiv$ $\left\{p \in S^{*}: \operatorname{dist}\left(p, S \backslash S^{*}\right)>\epsilon\right\}$. Choose a Riemannian metric $g_{S, \epsilon}$ on $M$ such that $J\left(T_{p} S\right) \perp T_{p} S$ for every $p \in S_{\epsilon}$ (see [Y1] for the simple construction). From now on we choose $g$ in the above discussion to be $g_{S, \epsilon}$ and let $V_{0}$ be the one-dimensional boundary subbundle $\left.\left.V\right|_{\partial F} \cap T S\right|_{\partial F}$. We denote the Maslov class $\mu\left(V, J, V_{0}\right)$ by $\mu\left(V, V_{0}\right)$. Note that it is independent of the choice of the Riemannian metric $g_{S, \epsilon}$.

For later reference, we insert a definition here.

Definition 2.4. Let $f: \Sigma \rightarrow M$ be a $J$-holomorphic curve. If $f$ is closed, then the Chern class $c(f)$ of $f$ is the first Chern class $c_{1}\left(f^{*}(T M, J)\right)$. If $f$ has its boundary on $S^{*}$ for a surface $S$, then the Maslov class $\mu(f)$ of $f$ is the Maslov class $\mu\left(f^{*}(T M, J),\left.f\right|_{\partial \Sigma} ^{*}(T S)\right)$. We set $\mu(f)$ to be $2 c(f)$ if $f$ is closed.

Next consider an embedded $J$-holomorphic curve $F$. Choose a normal bundle $V$ for $F$ as above. If $F$ has its boundary on $S^{*}$ for a surface $S$, then choose $V$ along with a boundary bundle $V_{0}$ as above. The "normal Maslov class" $\mu_{o}(F)$ of $F$ is $\mu\left(V, V_{0}\right)$ in this case. If $F$ is closed, then $\mu_{o}(F)$ is twice the "normal Chern class" $c_{o}(F)=c_{1}(V)=c_{1}(V, J)$.

Note that $c_{o}([f])$ and $\mu_{o}([f])$ are defined for $J$-holomorphic immersions $f$ in a similar way.

Next we choose a Riemannian metric $g_{1}$ such that $S$ is totally geodesic. Let $\exp$ denote the exponential map of $g_{1}$. Then $\exp (\varphi)$ is an embedded surface with boundary on $S$ for any sufficiently small $C^{1}$ section $\varphi$ of $V$ whose boundary values lie in $V_{0}$. Choose local coordinate frame fields $\frac{\partial}{\partial x_{1}}$ and $\frac{\partial}{\partial x_{2}}$ on $F$ such that the second is the image of the first under $J$. We set

$$
X=\frac{\partial}{\partial x_{1}} \exp (\varphi) \wedge \frac{\partial}{\partial x_{2}} \exp (\varphi)
$$

Then the oriented unit simple 2-vector field $w$ on $\exp (\varphi)$ is given by $w=X\left(|X|^{-1}\right)$.

Choose a small neighborhood $U$ of the zero section of $V$ such that $\left.\exp \right|_{U}$ is a diffeomorphism onto a neighborhood $U_{0}$ of $F$. Then it is easy to construct a smooth field of $J$-linear isomorphisms $\Theta$ on $U_{0}$, such that $\Theta_{p}$ maps $T_{p} M$ to $T_{\pi(v(p))} M$, where $p \in U, v(p)=\left.\exp \right|_{U} ^{-1}(p)$ and $\pi$ is the projection of $V$. Moreover, $\Theta_{p}=i d$ for $p \in F$. Indeed, we have $\Theta_{p}=\Theta_{p}^{o} \circ \tau_{p}$, where $\tau_{p}$ denotes the parallel transport from $p$ to $\pi(v(p))$ along the geodesic $\exp (t v(p))$, and $\Theta^{o}$ is some isomorphism of $T_{\pi(v(p))} M$ onto itself.

We set $H_{0}(\varphi)=\Theta\left(H(\exp (\varphi))\right.$, which is a section of the bundle $\left.\Lambda(M)\right|_{F}$. Note that $H_{0}(0)=0$. Let $\nabla$ be the connection of $g_{J}$. Replace $\varphi$ by $t \varphi$. By a simple 
computation we derive at $t=0$ :

$(2.1)$

$$
\begin{aligned}
\frac{\partial}{\partial t} H_{0}(t \varphi)= & \nabla_{\frac{\partial}{\partial t}} H(w)=J\left(\nabla_{\frac{\partial}{\partial x_{1}}} \varphi\right) \wedge \frac{\partial}{\partial x_{1}} \\
& -\frac{\partial}{\partial x_{2}} \wedge J\left(\nabla_{\frac{\partial}{\partial x_{2}}} \varphi\right)+\nabla_{\frac{\partial}{\partial x_{1}}} \varphi \wedge \frac{\partial}{\partial x_{2}}+\frac{\partial}{\partial x_{1}} \wedge \nabla_{\frac{\partial}{\partial x_{2}}} \varphi+a(\varphi),
\end{aligned}
$$

where $a$ is a certain field of linear maps (a tensor field). Choosing a local frame field $n, J n$ for $V$ and writing $\varphi=\varphi_{1} n+\varphi_{2} J n$, we then deduce (at $t=0$ )

$$
\begin{aligned}
\frac{\partial}{\partial t} H_{0}(t \varphi)= & \left(\frac{\partial \varphi_{1}}{\partial x_{1}}-\frac{\partial \varphi_{2}}{\partial x_{2}}\right)\left(n \wedge \frac{\partial}{\partial x_{2}}-J n \wedge J \frac{\partial}{\partial x_{2}}\right) \\
& +\left(\frac{\partial \varphi_{1}}{\partial x_{2}}+\frac{\partial \varphi_{2}}{\partial x_{1}}\right)\left(n \wedge \frac{\partial}{\partial x_{1}}-J n \wedge J \frac{\partial}{\partial x_{1}}\right)+a_{1}(\varphi),
\end{aligned}
$$

where $a_{1}$ is a section of the bundle $\left.V^{\prime} \otimes \Lambda(M)\right|_{F}$, with $V^{\prime}$ denoting the dual of $V$. This yields a linearization of the nonlinear operator $H$. A more convenient linearization can be obtained by using the isomorphism $\Phi$ given in Lemma 2.3. Choose a connection $\nabla^{o}$ on $V$. An easy computation using the above result then leads to $($ at $t=0)$

$$
\frac{\partial}{\partial t} \Phi\left(H_{0}(t \varphi)\right)=J \nabla^{o} \varphi-\nabla_{J}^{o} \varphi+a_{2}(\varphi)
$$

with $a_{2}$ denoting a section of the bundle $V^{\prime} \otimes \Lambda^{0,1}(V)$. Let $L(\varphi)$ denote the right hand side of this equation. $L$ is a Cauchy-Riemann operator with respect to the complex structure $J$ on $V$ and $T F$. $L$ acts e.g. between the Soblev spaces:

$$
L: W^{k, \sigma}\left(V, V_{0}\right) \rightarrow W^{k-1, \sigma}\left(\Lambda^{0,1}(V)\right)
$$

with $k \geq 1$ and $\sigma>1$. Let

$$
L^{*}: W^{k, \sigma}\left(\Lambda^{0,1}(V), V_{0}^{*}\right) \rightarrow W^{k-1, \sigma}\left(V, V_{0}\right)
$$

be the adjoint operator as introduced in $[\mathrm{MS}]$, where the adjoint boundary bundle $V_{0}^{*}$ is defined to be $\left\{\left.\eta \in \Lambda^{0,1}(V)\right|_{\partial F}: \eta(v) \in V_{0}\right.$ for all $\left.v \in T \partial F\right\}$. (The formulation in [MS] is slightly different, but applies directly here. One can also convert into that formulation easily.) Then $L^{*}$ is also a Cauchy-Riemann operator. From the results in $[\mathrm{MS}]$, the above deformation set-up and the implicit function theorem we deduce

Proposition 2.5. The index of $L$ is given by

$$
\text { ind } L=2-2 \gamma-l+\mu\left(V, V_{0}\right)=2-2 \gamma-l+\mu_{o}(F) \text {, }
$$

where $\gamma$ denotes the genus of $F$ and $l$ the number of boundary components of $F$. (If $F$ is closed, then $l=0$ and we replace $\mu\left(V, V_{0}\right)$ by $2 c_{1}(V)$, which is also $\mu_{o}(F)$ by the definition.) If $L$ is surjective (equivalently, $k e r L^{*}$ is trivial), then there is a $C^{1}$ neighborhood of $F$ such that the $J$-holomorphic curves (with boundary on $S$ ) in it constitute a smooth manifold $\mathcal{F}$ of dimension ind $L$. Indeed, coordinates for $\mathcal{F}$ are given by $F\left(t_{1}, \ldots, t_{k}\right)=\exp \left(t_{1} \varphi^{1}+\ldots+t_{k} \varphi^{k}+\varphi_{0}\left(t_{1}, \ldots, t_{k}\right)\right)$, where $k=$ ind $L$, $\left|t_{i}\right|<\varepsilon_{0}$ for a positive number $\varepsilon_{0}, \varphi^{1}, \ldots, \varphi^{k}$ are a base of $k e r L$, and $\varphi_{0}$ is orthogonal to $\operatorname{kerL}$ in $W^{0,2}$ with $\varphi(0)=0$.

Corollary. If $L$ is surjective, then $\mu_{o}(F) \geq 2 \gamma+l-2$. 
We also have

Proposition 2.6. Assume that $L$ is surjective and ind $L \geq 2$. Then for each $\varepsilon \in\left(0, \varepsilon_{0}\right]$, the union $\bigcup \mathcal{F}_{\varepsilon}$ of the members in $\mathcal{F}_{\varepsilon}$ contains an open set in the interior of $M$, where $\mathcal{F}_{\varepsilon}$ means the restriction of the $\mathcal{F}$ in Proposition 6.1 to parameters with absolute value not exceeding $\varepsilon$. Indeed, $\bigcup \mathcal{F}_{\varepsilon}$ contains a neighborhood of $F \backslash \varphi^{-1}(0)$ for any nontrivial element $\varphi$ of ker $L$.

Proof. To prove the first claim it suffices to show that for some $p \in \stackrel{\circ}{F}, \varphi^{1}(p)$ and $\varphi^{2}(p)$ are linearly independent. Choose a domain $\Omega$ in the interior of $F$ such that $\varphi^{1}$ has no zero in $\Omega$. Assume that there is a function $h$ on $\Omega$ such that (in $\Omega$ ) $\varphi^{2}=h \varphi^{1}$. Then $h$ is smooth. Since $L \varphi^{1}=0$ and $L \varphi^{2}=0$, one readily derives that the gradient of $h$ vanishes everywhere. Hence $h$ is a constant. Consequently, $\varphi^{2}-h \varphi^{1}$ is an element in $k e r L$ and vanishes in $\Omega$. Then $\varphi^{2}=h \varphi^{1}$, contradicting the fact that $\varphi^{1}$ and $\varphi^{2}$ are linearly independent. We conclude that $\varphi^{1}(p)$ and $\varphi^{2}(p)$ are linearly independent for some $p \in \Omega$. The second claim follows from the argument.

It is important to know when $L$ is surjective or $k e r L^{*}$ is trivial. The following proposition is the key here; it applies to an arbitary complex line bundle over a Riemann surface, a Cauchy-Riemann operator and a totally real boundary subbundle (if the Riemann surface has boundary).

Proposition 2.7. Assume that $\varphi \in$ ker $L$ is not identically zero. Then we have

$$
\mu\left(V, V_{0}\right)=2 m_{0}+m_{1}
$$

where $m_{0}$ denotes the number of interior zeros of $\varphi$ and $m_{1}$ the number of boundary zeros of $\varphi$, both counted with multiplicity. (If $F$ is closed, then $m_{1}=0$ and we replace $\mu\left(V, V_{0}\right)$ by $2 c_{1}(V)$. This case is due to Gromov [G].)

Proof. By the arguments in [Y1] for asymptotic behavior of pseudo holomorphic curves near branch points, one easily derives that $\varphi$ has isolated zeros. Moreover, if $z_{0}$ is a zero of $\varphi$, then

$$
\varphi(z)=\alpha\left(z-z_{0}\right)^{l}+O\left(\left|z-z_{0}\right|^{l+1}\right)
$$

for an integer $l \geq 1$ and a nonzero complex number $\alpha$. Here, we use suitable local coordinates $z=x_{1}+\sqrt{-1} x_{2}$ on $F$ and suitable local coordinates $w=\varphi_{1}+\sqrt{-1} \varphi_{2}$ for $\varphi$. Indeed, near a boundary zero, we choose the coordinates $\varphi_{1}$ and $\varphi_{2}$ such that $V_{0}$ is represented by $\varphi_{2}=0$.

We extend $F$ to a slighly larger surface $F_{\varepsilon}$. The complex vector bundle $V$ is then extended to $\tilde{V}$ over $F_{\varepsilon}$. We extend $\varphi$ by a suitable reflection across $\partial F$ to obtain a section $\tilde{\varphi}$ such that $\tilde{\varphi}$ has no zero in $F_{\varepsilon} \backslash F$ (cf. the proof of Lemma 5.2 in Section $5)$. Let $\tilde{V}_{0}$ denote the subbundle over $\partial F_{\varepsilon}$ generated by $\left.\tilde{\varphi}\right|_{\partial F_{\varepsilon}}$. By the asymptotic expansion (2.4) one readily derives

$$
\mu\left(\tilde{V}, \tilde{V}_{0}\right)=\mu\left(V, V_{0}\right)+m_{1}
$$

Now we choose loops of complex isomorphisms $\Phi_{p}: \tilde{V}_{p} \rightarrow \mathbb{C}, p \in \partial F_{\varepsilon}$, such that $\Phi_{p}\left(\left.\tilde{V}_{0}\right|_{p}\right)=\mathbb{R}$. Using $\Phi$, we glue the trivial bundle pairs $(B \times \mathbb{C}, \partial B \times \mathbb{R})$ to $\left(\tilde{V}, \tilde{V}_{0}\right)$ along each component of $\partial F_{\varepsilon}$. The resulting complex vector bundle over a closed surface is denoted by $\bar{V}$. The section $\tilde{\varphi}$ extends to a section $\bar{\varphi}$ of $\bar{V}$ such that the zeros of $\bar{\varphi}$ are the same as those of $\tilde{\varphi}$ or $\varphi$. The Euler class $e(\bar{V})$ of $\bar{V}$ is equal to $\frac{1}{2} \mu\left(\tilde{V}, \tilde{V}_{0}\right)$. This follows from the additivity of the Maslov class: the Maslov class 
$\mu(\bar{V})$ is the sum of $\mu\left(\tilde{V}, \tilde{V}_{0}\right)$ and several $\mu(B \times \mathbb{C}, \partial B \times \mathbb{R})$, but the former is equal to $2 c_{1}(\bar{V})=2 e(\bar{V})$ and the latter are zero. It can also be derived from a direct computation. On the other hand, $e(\bar{V})$ equals the number of zeros of $\bar{\varphi}$ counted with (signed) multiplicities. We deduce that

$$
\mu\left(\tilde{V}, \tilde{V}_{0}\right)=2 m_{0}+2 m_{1} \text {. }
$$

The desired formula follows from (2.5) and (2.6). Alternatively, we can glue a copy of $\left(\tilde{V}, \tilde{V}_{0}\right)$ to itself and argue in the same way.

Lemma 2.8. The Maslov class $\mu\left(\Lambda^{0,1}(V), V_{0}^{*}\right)$ is equal to $-4+4 \gamma+2 l-\mu\left(V, V_{0}\right)$.

Proof. This formula can be proven by a direct computation. An easy way to see it is as follows. On the one hand, the index of $L^{*}$ is given by $2-2 \gamma-l+\mu\left(\Lambda^{0,1}(V), V_{0}^{*}\right)$. On the other hand, it is equal to - ind $L$. The result follows.

Theorem 2.9. 1) If $\mu\left(\Lambda^{0,1}(V), V_{0}^{*}\right)<0$, then ker $L^{*}=\{0\}$ and hence $L$ is surjective. Consequently, $L$ is surjective provided that $\mu\left(V, V_{0}\right)>-4+4 \gamma+2 l$.

2) If $\mu\left(V, V_{0}\right)=0$, then $\operatorname{dim} \operatorname{ker} L=1$. Moreover, if $\varphi \in \operatorname{kerL}$ and is not identically zero, then $\varphi$ has no zeros.

(If $F$ is closed, then $l=0$ and we replace $\mu\left(V, V_{0}\right)$ by $2 c_{1}(V)$ and $\mu\left(\Lambda^{0,1}(V), V_{0}^{*}\right)$ by $2 c_{1}\left(\Lambda^{0,1}(V)\right)$.)

This is an immediate consequence of Proposition 2.7 and Lemma 2.8. Note that this theorem applies in the generality mentioned for Proposition 2.7.

To have a complete picture, we would also like to consider deformations of parametrized $J$-holomorphic curves (which are not necessarily embedded or immersed). The nonlinear Cauchy-Riemann operator is simply $J \circ d f \circ j+d f$, where $f$ denotes a map from a Riemann surface $\Sigma$ with almost complex structure $j$. The linearized Cauchy-Riemann operator $L_{0}: W^{k, \sigma}\left(f^{*} T M\right) \rightarrow W^{k-1, \sigma}\left(\Sigma \otimes f^{*} T M\right)$ at a $J$-holomorphic curve $f$ is naturally defined; see [M4] or [MS]. For a Riemann surface $(\Sigma, j)$, let $\mu(\Sigma)$ denote the Maslov class of the bundle pair $(T \Sigma, T \partial \Sigma)$. It is easy to see that $\mu(\Sigma)=4-4 \gamma-2 l$, where $\gamma$ denotes the genus of $\Sigma$ and $l$ the number of its boundary components. If $\Sigma$ is closed, then $\mu(\Sigma)$ means twice the Euler class of $\Sigma$.

Theorem 2.10. Let $f: \Sigma \rightarrow M$ be J-holomorphic such that $f(\partial \Sigma) \subset S$ in case $\partial \Sigma$ is nonempty. If $f$ is immersed, then the linearized Cauchy-Riemann operator $L_{0}$ along $f$ is surjective if and only if $\mu(f)>0$.

The "if" part follows from Theorem 2.9 and a simple decomposition argument for the operator $L_{0}$ (one decomposes $L_{0}$ into the tangential and normal components). The "only if" part is contained in Lemma 3.7 and Proposition 3.8 below.

\section{Generic Regularity}

In this section we first consider the effect of perturbing $J$ or $S$ on the surjectivity of the linearized Cauchy-Riemann operator $L$ introduced in the last section. Note that the set-up for $L$ depends only on the choice of the Riemannian metrics $g_{S, \epsilon}$ and $g_{1}$. Such a choice will be called a set-up choice.

Definition 3.1. Let $S$ be given. $J$ is called "(rationally) $S$-regular in the embedding set-up" if for some set-up choice, the operator $L$ is surjective for an arbitary embedded $J$-holomorphic curve (disk) $F$ with boundary on $S^{*}$. 
Let $\mathcal{J}$ denote the space of smooth almost complex structures on $M$. For a given $J_{0}$, set $\mathcal{J}_{J_{0}}=\left\{J \in \mathcal{J}:\left.J\right|_{\partial M}=\left.J_{0}\right|_{\partial M}\right\}$. For a given symplectic form $\omega$, set $\mathcal{J}_{\omega}=\{J \in \mathcal{J}: J$ is uniformly tamed by $\omega\}$. For $J \in \mathcal{J}_{\omega}$ we denote by $g_{\omega, J}$ the symmetrization of $\omega(\cdot, J)$. We introduce a topology on $\mathcal{J}_{\omega}$ in the following way. For each $J \in \mathcal{J}_{\omega}$, set $\mathcal{X}_{J}=\{K: K$ is a smooth section of End(TM) such that $J K+K J=0$, and $\|K\|<\infty\}$ with

$$
\|K\|=\|K\|_{C^{1}(M)}+\sum_{k=1}^{\infty} \varepsilon_{k}\|K\|_{C^{k}\left(\Omega_{k}\right)},
$$

where the $\Omega_{k}$ are an increasing sequence of compact sets of $M$ whose union is $M$, the $\varepsilon_{k}$ are a sufficiently rapidly decreasing sequence of positive numbers, and the $C^{k}$ norms are defined in terms of the metric $g_{\omega, J}$. This is a direct generalization of the smooth norm of Floer $[\mathrm{F}][\mathrm{MS}] . \mathcal{X}_{J}$ is a separable Banach space and dense in the space of $W^{0,1}$-sections $K$ of $\operatorname{End}(T M)$ with $J K+K J=0$ (cf. [MS]). There is a natural map $\Theta: \mathcal{B}_{1} \equiv\left\{K \in \mathcal{X}_{J}:\|K\|<1\right\} \rightarrow \mathcal{J}_{\omega}$,

$$
\Theta(K)=J \circ(I d+K) \circ(I d-K)^{-1} ;
$$

see $[\mathrm{A}]$. We set $\mathcal{U}_{J}=\Theta\left(\mathcal{B}_{1}\right)$ and equip it with the topology induced by $\Theta$.

Proposition 3.2. Let $\omega$ and a closed surface $S$ with isolated complex points be given. For each $J_{1} \in \mathcal{J}_{\omega}$, the set of rationally $S$-regular (in the embedding set-up) $J$ in $\mathcal{U}_{J_{1}}$ is a countable intersection of open and dense sets. If $\partial M$ is $J_{0}$-convex for some $J_{0}$, then the set of rationally $S$-regular (in the embedding set-up) $J$ in $\mathcal{U}_{J_{1}} \cap \mathcal{J}_{J_{0}}$ is a countable intersection of open and dense sets for every $J_{1} \in \mathcal{J}_{J_{0}} \cap \mathcal{J}_{\omega}$. The same holds if "rationally S-regular" is replaced by "S-regular". For convenience, e.g. in the first case, we shall simply say (by abuse of terminology) that the set of rationally $S$-regular $J$ is a countable intersection of open and dense sets in $\mathcal{J}_{\omega}$. We shall adopt this convention also in other similar contexts below.

Remark. A similar result holds without $\omega$.

The proof is similar to the proof of Proposition 3.6 below; we leave the details to the reader. To deal with perturbations of (compact) $S$ with a fixed $J$, we introduce a topology on the space of smooth $S$ in a way similar to (3.1), replacing $\Omega_{k}$ by $S$ for all $k$.

Theorem 3.3. Assume that $\partial M$ is $J$-convex. Let $\mathcal{S}$ be an isotopy class of $S \subset$ $\partial M$ which have isolated complex points. For each given $J$, there is a countable intersection of open and dense sets of $S$ in $\mathcal{S}$ such that $J$ is (rationally) $S$-regular in the embedding set-up.

Proof. We consider the space $\mathcal{N}$ of pairs $(F, S)$ with $\partial F \in S^{*}$, where $S$ belongs to $\mathcal{S}$ and $F$ belongs to a given isotopy class $A$. For a small positive number $\epsilon$, set $\mathcal{N}_{\epsilon}=\left\{(F, S) \in \mathcal{N}: \operatorname{diam}_{g_{1}} S>2 \epsilon\right.$, dist $\left._{g_{1}}\left(\partial F, S \backslash S^{*}\right)>\epsilon\right\}$, where $g_{1}$ is some metric. Let $\pi$ be the projection to the second factor, i.e. $\pi((F, S))=S$. We define a Banach manifold struture on $\mathcal{N}_{\epsilon}$ as follows. Consider $(F, S) \in \mathcal{N}_{\epsilon}$ and a sufficiently small neighborhood $\mathcal{U}$ of $(F, S)$. An element $S^{\prime}$ of $\pi(\mathcal{U})$ can be written as $\exp \left(\varphi_{S^{\prime}}\right)$ for a small section $\varphi_{S^{\prime}}$ of $V_{S}$, where the normal bundle $V_{S}$ of $S$ in $\partial M$ and the exponential map are both defined in terms of a metric $g_{0}$ under which $S_{0}$ and $\partial M$ are totally geodesic.

To proceed, choose a continuous linear extension map $\alpha$ which extends sections of $\left.T M\right|_{\partial F}$ to sections of $\left.T M\right|_{F}$. Then we set $F_{S^{\prime}}=\operatorname{image}\left(f_{S^{\prime}}\right)$, where $f_{S^{\prime}}=$ 
$\exp \left(\alpha\left(\left.\varphi_{S^{\prime}}\right|_{F}\right)\right)$. For each $S^{\prime}$, choose a metric $g_{S^{\prime}}$ such that $S^{\prime}$ and $\partial M$ are totally geodesic, and let $\exp _{S^{\prime}}$ be its exponential map. We can choose $g_{S^{\prime}}$ such that it depends smoothly on $S^{\prime}$ and $g_{S}=g_{0}$. For an elment $\left(F^{\prime}, S^{\prime}\right) \in \mathcal{U}$ we can then represent $F^{\prime}$ as $\exp _{S^{\prime}}\left(\varphi_{F^{\prime}}\right)$ for a section $\varphi_{F^{\prime}}$ of the normal bundle $V\left(F_{S^{\prime}}\right)$ of $F_{S^{\prime}}$ which is defined in the same way as the normal bundle $V$ of $F$ in the last section. (In particular, $V(F)=V$.) Note that we can choose the metric $g_{S^{\prime}, \epsilon}$ in a smooth way. Now we choose a complex linear isomorphism $\Upsilon_{S^{\prime}}: V\left(F_{S^{\prime}}\right) \rightarrow V$, such that it depends smoothly on $S^{\prime}$. We set

$$
\Pi\left(F^{\prime}, S^{\prime}\right)=\left(\Upsilon_{S^{\prime}}\left(\varphi_{F^{\prime}}\right), S\right) .
$$

Along with the Banach manifold structure of $\mathcal{S}$, this induces a Banach manifold structure on $\mathcal{N}_{\epsilon}$ (restricting to sections $\varphi_{F^{\prime}}$ in the Sobolev space $W^{k, \sigma}$ for a large $k)$.

The nonlinear Cauchy-Riemann operator $H$ extends naturally to $\mathcal{N}_{\epsilon}$. The auxiliary isomorphisms $\Theta$ can also be extended by using the parallel transport along the geodesics $\exp (\alpha(t \varphi(p))), p \in F$ (composed with suitable auxiliary isomorphisms at $p)$ and the corresponding auxiliary isomorphisms for $F_{S^{\prime}}$. Then we obtain a linearization $\tilde{L}$ of $H$ at any given $(F, S)$ with $F$ being $J$-holomorphic, which acts between the tangent space $\left.T\right|_{(F, S)} \mathcal{N}_{\epsilon}$ and $W^{k-1, \sigma}\left(\Lambda^{0,1}(V)\right)$. A subspace of $\left.T\right|_{(F, S)} \mathcal{N}_{\epsilon}$ can be identified with the space $W^{k, \sigma}\left(V, V_{0}\right)$; for elements $\varphi$ of this space we have

$$
\tilde{L} \varphi=L \varphi
$$

where $L$ is the linearized Cauchy-Riemann operator introduced in the last section. We claim that the image of $\tilde{L}$ is dense. Let $\psi$ be an element in a suitable dual function space which annilates image $(\tilde{L})$ (see $[\mathrm{MS}]$ for details). In particualar, $\psi$ annilates the image of $W^{k, \sigma}\left(V, V_{0}\right)$ under $L$. By the basic linear theory [MS], $\psi$ must be a smooth solution of the equation $L^{*} \psi=0$ with boundary values in $V_{0}^{*}$. Now consider an arbitary section $\varphi$ of $\left.V\right|_{\partial F}$. It is clear that there is a smooth path $\left(F_{t}, S_{t}\right)$ in $\mathcal{U}$ with $F_{t}=[\exp (\alpha(t \varphi))]$ and $S_{0}=S$. Let $\tilde{\varphi}$ denote the initial tangent vector of this path. Then we have

$$
\left.\tilde{L} \tilde{\varphi} \equiv \frac{\partial}{\partial t} \Phi\left(H_{0}(t \alpha(\varphi))\right)\right|_{t=0}=L \alpha(\varphi) .
$$

It follows that $\psi$ annilates $L \alpha(\varphi)$. Since $\psi$ satisfies the equation $L^{*} \psi=0$ and the boundary values of $\alpha(\varphi)$ are arbitary, one easily deduces that the boundary values of $\psi$ vanish identically. The boundary asymtotical analysis in [Y1] (as has been used earlier) then implies that $\psi$ vanishes everywhere. Since $\psi$ is arbitary, $\tilde{L}$ has dense image.

Since a restriction of $\tilde{L}$ is the Fredholm operator

$$
L: W^{k, \sigma}\left(V, V_{0}\right) \rightarrow W^{k-1, \sigma}\left(\Lambda^{0,1}(V)\right),
$$

it is easy to see that the density of image $(\tilde{L})$ implies that $\tilde{L}$ is surjective and has a right inverse. (However, $\tilde{L}$ itself is not Fredholm. Its kernel has infinite dimensions.) Since $(F, S)$ is arbitary, it follows from the implicit function theorem that the "big moduli space" $H^{-1}(0)$ is a manifold. It is then easy to see that the projection $\pi$ is Fredholm. Applying the Sard-Smale transversality theorem as in [MS] or [M4], we then deduce that there is a countable intersection $\mathcal{S}_{\epsilon}^{*}$ of open and dense sets in $\mathcal{S}_{\epsilon}=\left\{S \in \mathcal{S}: \operatorname{diam}_{g_{1}} S>2 \epsilon\right\}$ such that for $S \in \mathcal{S}_{\epsilon}^{*}$ the following holds: for every $J$-holomorphic $F$ in $\pi^{-1}(S)$, the operator $L: W^{k, \sigma}\left(V, V_{0}\right) \rightarrow W^{k-1, \sigma}\left(\Lambda^{0,1}(V)\right)$ is surjective. Choosing a sequence of $\epsilon$ and using the argument in the proof of 
Proposition 3.6 below to deal with all isotopy classes $A$, we then arrive at the conclusion of the proposition.

We also need to consider deformations of parametrized $J$-holomorphic spheres. The theory here is well-developped [M4] [MS]. The nonlinear Cauchy-Riemann operator is simply $J \circ d f \circ j+d f$, where $f$ denotes a map from the sphere $\mathbb{S}^{2}$ and $j$ the almost complex structure of the sphere. The linearized Cauchy-Riemann operator $L_{0}: W^{k, \sigma}\left(f^{*} T M\right) \rightarrow W^{k-1, \sigma}\left(T^{0,1} S^{2} \otimes f^{*} T M\right)$ at a $J$-holomorphic sphere $f$ is naturally defined; see [M4] or [MS].

Definition 3.4. A $J$-holomorphic sphere $f$ is called "simple" if it does not factor through holomorphic branched coverings of $\mathbb{S}^{2}$, or equivalently, $f^{-1}(f(z))=\{z\}$ for some $z \in \mathbb{S}^{2}$.

Definition 3.5. Let $A$ be a homotopy class of spheres in $M . J$ is called " $A$-regular" if the above linearized Cauchy-Riemann operator $L_{0}$ is surjective for every simple $J$-holomorphic sphere $f \in A$. $J$ is called "rationally regular" if it is $A$-regular for every $A$.

Proposition 3.6. The set of rationally regular $J$ in $\mathcal{J}_{\omega}$ or $\mathcal{J}_{\omega} \cap \mathcal{J}_{J_{0}}$ is a countable intersection of open and dense sets.

Proof. We only treat the first case. The second case is similar. First assume that $M$ is compact. By e.g. [MS], for each homotopy class $A$, the set of $A$-regular $J$ in $\mathcal{J}_{\omega}$ is a countable intersection of open and dense subsets of $\mathcal{J}_{\omega}$. Gromov's compactness theorem (see [Y1]) implies that for each $a>0$, there are only finitely many homotopy classes $A$ with $\omega(A) \leq a$ which contain $J$-holomorphic spheres. Hence the set of rationally regular $J$ in $\mathcal{J}_{\omega}$ is a countable intersection of open and dense sets. If $M$ is non-compact, we represent it as a countable union of increasing compact domains and restrict to $J$-holomorphic spheres contained in them.

The following fact is well-known:

Lemma 3.7. If $f$ is $J$-regular, i.e. $L_{0}$ is surjective at $f$, then $c(f) \geq 1$.

Proof. The index of $L_{0}$ is given by $4+2 c(f)$ [G] [MS]. If $L_{0}$ is surjective, then this is equal to $\operatorname{ker} L_{0}$. But dim $\operatorname{ker} L_{0} \geq 6$, because the dimension of the automorphism group of $\mathbb{S}^{2}$ is 6 .

One can formulate a similar deformation set-up for parametrized $J$-holomorphic curves with boundary. The notion of "(rationally) $S$-regular in the parametric setup" is defined in a natural way; we leave the details to the reader. The above arguments for spheres lead to (note that the arguments in [M4] and [MS] apply directly to disks)

Proposition 3.8. 1) For a given $S \subset \partial M$ (and $J_{0}$ ) the set of (rationally) S-regular $J$ in $\mathcal{J}_{\omega}$ or $\mathcal{J}_{\omega} \cap \mathcal{J}_{J_{0}}$ is a countable intersection of open and dense sets.

2) If a J-holomorphic disk $f$ is $J$-regular, then $\mu(f) \geq 1$.

We also have a result similar to Theorem 3.3:

Proposition 3.9. Let $\mathcal{S}$ be an isotopy class of $S \subset M$ which have isolated complex points. For each given $J$, there is a countable intersection of open and dense sets of $S$ in $\mathcal{S}$ such that $J$ is (rationally) $S$-regular in the parametric set-up.

Note that unlike Theorem 3.3, here one cannot restrict to $S \subset \partial M$. 


\section{Deformation OF NEARLY $J$-HOLOMORPhiC DiskS}

Our goal in this section is to prove the existence part of Theorem 1. Consider an elliptic complex point $p_{0} \in S$ and choose preferred coordinates around $p_{0}$ such that $a=d=0$. Performing (1.4) with $\alpha_{1}=-1, \alpha=1$ and $\beta=\beta_{1}=0$ if necessary, we can in addition assume $b>0$ and $c<0$. Set $\lambda_{1}=-c$ and $\lambda_{2}=b$. Then the quadratic form $Q\left(x_{1}, x_{2}\right)=\frac{1}{2} \lambda_{1} x_{1}^{2}+\frac{1}{2} \lambda_{2} x_{2}^{2}$ is positive definite. We set $\Omega=\{Q \leq 1\}$.

Let $U$ be the euclidean domain of the chosen preferred coordinates. We consider the dilation $T_{R}(x)=R x$ for $R>0$. Set $U_{R}=T_{R}^{-1}(U)$ and let $J_{R}$ be the almost complex structure on $U_{R}$ induced from $J$. Then

$$
\left(J_{R}\right)_{x} \frac{\partial}{\partial x_{i}}=\sum_{j=1}^{4} J_{i j}(R x) \frac{\partial}{\partial x_{j}}, \quad 1 \leq i \leq 4 .
$$

We are going to construct $J_{R}$-holomorphic disks with boundary on the $\left(x_{1}, x_{2}\right)$ plane, because they yield the desired $J$-holomorphic disks through the dilation $T_{R}$.

For small $R$, we introduce initial disks $f^{0}: \Omega \rightarrow U_{R}$ with

$$
f_{1}^{0}=x_{1}, f_{2}^{0}=x_{2}, f_{3}^{0}=R(Q-1) \text { and } f_{4}^{0}=0 .
$$

These disks are embedded. We have the following tangent vector fields for $f^{0}$ :

$$
\begin{aligned}
& v_{1}^{0}=e_{1}+R \lambda_{1} x_{1} e_{3}, \\
& v_{2}^{0}=e_{2}+R \lambda_{2} x_{2} e_{3},
\end{aligned}
$$

and transversal vector fields

$$
\begin{aligned}
& n_{1}=\lambda_{1} x_{1} e_{1}+\lambda_{2} x_{2} e_{2}+R(Q-1) e_{3}, \\
& n_{2}=e_{4} .
\end{aligned}
$$

Here $e_{1}=\frac{\partial}{\partial x_{1}}, e_{2}=\frac{\partial}{\partial x_{2}}, e_{3}=\frac{\partial}{\partial x_{3}}$ and $e_{4}=\frac{\partial}{\partial x_{4}}$.

The disks $f^{0}$ are nearly $J_{R}$-holomorphic, but we need not check it at this stage. Our goal is to deform $f^{0}$ into $J_{R}$-holomorphic. The deformation set-up in the last section can also be applied to nearly $J$-holomorphic disks. However, since the $\left(x_{1}, x_{2}\right)$-plane becomes complex in the limit as $R \rightarrow 0$, the boundary conditions in the set-up are very delicate. Moreover, it is not very handy for computations. We shall adopt a different approach. Consider an embedded disk $F$ in $M$. Choose a tangent frame field $v, \tilde{v}$ and a vector field $n$ along $F$ which is non-tangential to $F$ everywhere. We have

Lemma 4.1. $F$ is J-holomorphic if and only if the following equations hold:

$$
\begin{aligned}
& J v \wedge v \wedge \tilde{v} \wedge n=0, \\
& J \tilde{v} \wedge v \wedge \tilde{v} \wedge n=0 .
\end{aligned}
$$

Proof. It is easy to see that (4.1) and (4.2) imply

$$
\begin{aligned}
& J v=a_{1} v+b_{1} \tilde{v}+c_{1} n, \\
& J \tilde{v}=a_{2} v+b_{2} \tilde{v}+c_{2} n .
\end{aligned}
$$

If either $c_{1}$ or $c_{2}$ is not zero at a point, then the subspace spanned by $v, \tilde{v}$ and $n$ at that point would be invariant under $J$. This is impossible. Hence (4.1) and (4.2) imply that $F$ is $J$-holomorphic. On the other hand, the $J$-holomorphic property clearly implies (4.1) and (4.2). 
For $C^{1}$ functions $\varphi_{1}, \varphi_{2}$ on $\Omega$ with $\left.\varphi_{2}\right|_{\partial \Omega}=0$ we define a disk $f$ by setting

$$
\begin{aligned}
& f_{1}=x_{1}\left(1+\lambda_{1} \varphi_{1}\right), \quad f_{2}=x_{2}\left(1+\lambda_{2} \varphi_{1}\right), \\
& f_{3}=R(Q-1)\left(1+\varphi_{1}\right), \quad f_{4}=R \varphi_{2} .
\end{aligned}
$$

If $R, \varphi_{1}$ and $\varphi_{2}$ are sufficiently small, the disk $f$ is also embedded. We obtain its tangent vector fields

$$
\begin{aligned}
& v_{1}=\left(1+\lambda_{1} \varphi_{1}+\lambda_{1} x_{1} \frac{\partial \varphi_{1}}{\partial x_{1}}\right) e_{1}+\lambda_{2} x_{2} \frac{\partial \varphi_{1}}{\partial x_{1}} e_{2} \\
& +R\left[(Q-1) \frac{\partial \varphi_{1}}{\partial x_{1}}+\lambda_{1} x_{1}\left(1+\varphi_{1}\right)\right] e_{3}+R \frac{\partial \varphi_{2}}{\partial x_{1}} e_{4}, \\
& v_{2}=\lambda_{1} x_{1} \frac{\partial \varphi_{1}}{\partial x_{2}} e_{1}+\left(1+\lambda_{2} \varphi_{1}+\lambda_{2} x_{2} \frac{\partial \varphi_{1}}{\partial x_{2}}\right) e_{2} \\
& +R\left[(Q-1) \frac{\partial \varphi_{1}}{\partial x_{2}}+\left(\lambda_{2} x_{2}\left(1+\varphi_{1}\right)\right] e_{3}+R \frac{\partial \varphi_{2}}{\partial x_{2}} e_{4} .\right.
\end{aligned}
$$

By Lemma 4.1, image $(f)$ is $J_{R}$-holomorphic if and only if the following equations hold:

$$
\begin{aligned}
& J_{R} v_{1} \wedge v_{1} \wedge v_{2} \wedge n_{2}=0 \\
& J_{R} v_{2} \wedge v_{1} \wedge v_{2} \wedge n_{2}=0
\end{aligned}
$$

assuming that $R$ is small enough for $n_{2}$ to be nontangential to $f$ everywhere.

We compute

$$
\begin{aligned}
v_{1} \wedge v_{2} \wedge n_{2}= & L_{12} e_{1} \wedge e_{2} \wedge e_{4}+L_{13} e_{1} \wedge e_{3} \wedge e_{4} \\
& +L_{23} e_{2} \wedge e_{3} \wedge e_{4},
\end{aligned}
$$

where

$$
\begin{aligned}
L_{12}= & 1+\left(\lambda_{1}+\lambda_{2}\right) \varphi_{1}+\lambda_{1} x_{1} \frac{\partial \varphi_{1}}{\partial x_{1}}+\lambda_{2} x_{2} \frac{\partial \varphi_{1}}{\partial x_{2}} \\
& +\lambda_{1} \lambda_{2} \varphi_{1}^{2}+\lambda_{1} \lambda_{2} x_{1} \varphi_{1} \frac{\partial \varphi_{1}}{\partial x_{1}}+\lambda_{1} \lambda_{2} x_{2} \varphi_{1} \frac{\partial \varphi_{1}}{\partial x_{2}} \\
L_{13}= & R \lambda_{2} x_{2}+R \lambda_{2} x_{2}\left(1+\lambda_{1}\right) \varphi_{1} \\
& +R \lambda_{1} \lambda_{2} x_{1} x_{2} \frac{\partial \varphi_{1}}{\partial x_{1}}-R\left[\lambda_{1}^{2} x_{1}^{2}+(1-Q)\right] \frac{\partial \varphi_{1}}{\partial x_{2}} \\
& +R\left(\lambda_{1} \lambda_{2} x_{2} \varphi_{1}^{2}+\lambda_{1}(Q-1) \varphi_{1} \frac{\partial \varphi_{1}}{\partial x_{2}}-\lambda_{1}^{2} x_{1}^{2} \varphi_{1} \frac{\partial \varphi_{1}}{\partial x_{2}}\right), \\
L_{23}=- & R \lambda_{1} x_{1}-R \lambda_{1} x_{1}\left(1+\lambda_{2}\right) \varphi_{1}+R\left[\lambda_{2}^{2} x_{2}^{2}+(1-Q)\right] \frac{\partial \varphi_{1}}{\partial x_{1}} \\
- & R \lambda_{1} \lambda_{2} x_{1} x_{2} \frac{\partial \varphi_{1}}{\partial x_{2}}+R\left[\lambda_{2}^{2} x_{2}^{2} \varphi_{1} \frac{\partial \varphi_{1}}{\partial x_{1}}+(1-Q) \lambda_{2} \varphi_{1} \frac{\partial \varphi_{1}}{\partial x_{1}}\right. \\
- & \left.\lambda_{1} \lambda_{2} x_{1} x_{2} \varphi_{1} \frac{\partial \varphi_{1}}{\partial x_{2}}\right] .
\end{aligned}
$$


It follows that

$$
\begin{aligned}
J_{R} v_{1} \wedge v_{1} \wedge v_{2} \wedge n_{2}= & \left(1+\lambda_{1} \varphi_{1}+\lambda_{1} x_{1} \frac{\partial \varphi_{1}}{\partial x_{1}}\right)\left(J_{13} L_{12}-J_{12} L_{13}+J_{11} L_{23}\right) \\
& \lambda_{2} x_{2} \frac{\partial \varphi_{1}}{\partial x_{1}}\left(J_{23} L_{12}-J_{22} L_{13}+J_{21} L_{23}\right) \\
& R\left[(Q-1) \frac{\partial \varphi_{1}}{\partial x_{1}}+\left(1+\varphi_{1}\right) \lambda_{1} x_{1}\right]\left(J_{33} L_{12}-J_{32} L_{13}+J_{31} L_{23}\right) \\
& R \frac{\partial \varphi_{2}}{\partial x_{1}}\left(J_{43} L_{12}-J_{42} L_{13}+J_{41} L_{23}\right),
\end{aligned}
$$

and

$$
\begin{aligned}
J_{R} v_{2} \wedge v_{1} \wedge v_{2} \wedge n_{2}= & \lambda_{1} x_{1} \frac{\partial \varphi_{1}}{\partial x_{2}}\left(J_{13} L_{12}-J_{12} L_{13}+J_{11} L_{23}\right) \\
& +\left(1+\lambda_{2} \varphi_{1}+\lambda_{2} x_{2} \frac{\partial \varphi_{1}}{\partial x_{2}}\right)\left(J_{23} L_{12}-J_{22} L_{13}+J_{21} L_{23}\right) \\
& +R\left[(Q-1) \frac{\partial \varphi_{1}}{\partial x_{2}}+\lambda_{2} x_{2}\left(1+\varphi_{1}\right)\right]\left(J_{33} L_{12}-J_{32} L_{13}+J_{31} L_{23}\right) \\
& +R \frac{\partial \varphi_{2}}{\partial x_{2}}\left(J_{43} L_{12}-J_{42} L_{13}+J_{41} L_{23}\right),
\end{aligned}
$$

where $J_{i j}$ stands for $J_{i j}(R f)$. We have

$$
\left\{\begin{array}{l}
J_{11}=R A_{11}\left(x_{1}, x_{2}, \varphi_{1}, \varphi_{2}, R\right) \\
J_{12}=1+R A_{12}\left(x_{1}, x_{2}, \varphi_{1}, \varphi_{2}, R\right) \\
J_{13}=R \lambda_{2} x_{2}\left(1+\lambda_{2} \varphi_{1}\right)+R^{2} A_{13}\left(x_{1}, x_{2}, \varphi_{1}, \varphi_{2}, R\right) \\
J_{21}=-1+R A_{21}\left(x_{1}, x_{2}, \varphi_{1}, \varphi_{2}, R\right) \\
J_{22}=R A_{22}\left(x_{1}, x_{2}, \varphi_{1}, \varphi_{2}, R\right) \\
J_{23}=-R \lambda_{1} x_{1}\left(1+\lambda_{1} \varphi_{1}\right)+R^{2} A_{23}\left(x_{1}, x_{2}, \varphi_{1}, \varphi_{2}, R\right) \\
J_{31}=R A_{31}\left(x_{1}, x_{2}, \varphi_{1}, \varphi_{2}, R\right) \\
J_{32}=R A_{32}\left(x_{1}, x_{2}, \varphi_{1}, \varphi_{2}, R\right) \\
J_{33}=R A_{33}\left(x_{1}, x_{2}, \varphi_{1}, \varphi_{2}, R\right) \\
J_{41}=R A_{41}\left(x_{1}, x_{2}, \varphi_{1}, \varphi_{2}, R\right) \\
J_{42}=R A_{42}\left(x_{1}, x_{2}, \varphi_{1}, \varphi_{2}, R\right) \\
J_{43}=-1+R A_{43}\left(x_{1}, x_{2}, \varphi_{1}, \varphi_{2}, R\right)
\end{array}\right.
$$

where $A_{i j}$ are smooth functions of their arguments. These formulas easily follow from the properties of $J_{i j}$ which were given in section 1 .

We deduce that

$$
\begin{aligned}
J_{13} L_{12}-J_{12} L_{13}+J_{11} L_{23}= & R \lambda_{2} x_{2}\left(\lambda_{2}-1\right) \varphi_{1} \\
& +R\left[\lambda_{1}^{2} x_{1}^{2}+\lambda_{2}^{2} x_{2}^{2}+(1-Q)\right] \frac{\partial \varphi_{1}}{\partial x_{2}} \\
& +R C_{1}\left(x_{1}, x_{2}, \varphi_{1}, \frac{\partial \varphi_{1}}{\partial x_{1}}, \frac{\partial \varphi_{1}}{\partial x_{2}}, R\right) \\
& +R^{2} A_{1}\left(x_{1}, x_{2}, \varphi_{1}, \varphi_{2}, \frac{\partial \varphi_{1}}{\partial x_{1}}, \frac{\partial \varphi_{1}}{\partial x_{2}}, R\right),
\end{aligned}
$$




$$
\begin{aligned}
& J_{23} L_{12}-J_{22} L_{13}+J_{21} L_{23}= R \lambda_{1}\left(1-\lambda_{1}\right) x_{1} \varphi_{1}-R\left[\lambda_{1}^{2} x_{1}^{2}+\lambda_{2}^{2} x_{2}^{2}\right. \\
&+(1-Q)] \frac{\partial \varphi_{1}}{\partial x_{1}}+R C_{2}\left(x_{1}, x_{2}, \varphi_{1}, \frac{\partial \varphi_{1}}{\partial x_{1}}, \frac{\partial \varphi_{1}}{\partial x_{2}}\right) \\
&+ R^{2} A_{2}\left(x_{1}, x_{2}, \varphi_{1}, \varphi_{2}, \frac{\partial \varphi_{1}}{\partial x_{1}}, \frac{\partial \varphi_{1}}{\partial x_{2}}, R\right), \\
&(4.16) \quad J_{33} L_{12}-J_{32} L_{13}+J_{31} L_{23}=R A_{3}\left(x_{1}, x_{2}, \varphi_{1}, \varphi_{2}, \frac{\partial \varphi_{1}}{\partial x_{1}}, \frac{\partial \varphi_{1}}{\partial x_{2}}, R\right), \\
& J_{43} L_{12}-J_{42} L_{13}+J_{41} L_{23}=-1-\left(\lambda_{1}+\lambda_{2}\right) \varphi_{1}-\lambda_{1} x_{1} \frac{\partial \varphi_{1}}{\partial x_{1}}-\lambda_{2} x_{2} \frac{\partial \varphi_{1}}{\partial x_{2}} \\
&+R A_{4}\left(x_{1}, x_{2}, \varphi_{1}, \varphi_{2}, \frac{\partial \varphi_{1}}{\partial x_{1}}, \frac{\partial \varphi_{1}}{\partial x_{2}}, R\right), \\
&+C_{4}\left(x_{1}, x_{2}, \varphi_{1}, \frac{\partial \varphi_{1}}{\partial x_{1}}, \frac{\partial \varphi_{1}}{\partial x_{2}}\right)
\end{aligned}
$$

where $A_{i}$ are smooth functions and $C_{i}$ are quadratic forms in $\varphi, \frac{\partial \varphi_{1}}{\partial x_{1}}, \frac{\partial \varphi_{1}}{\partial x_{2}}$ with smooth functions of $x_{1}, x_{2}$ and $R$ as coefficients.

Now it is easy to derive from the equations (4.12) (4.13), (4.15), (4.16), (4.17) and (4.18) the following lemma.

Lemma 4.2. The equations (4.6) and (4.7), after being divided by $R$, can be written in the following way:

$$
\begin{aligned}
& {\left[\lambda_{1}^{2} x_{1}^{2}+\lambda_{2}^{2} x_{2}^{2}+(1-Q)\right] \frac{\partial \varphi_{1}}{\partial x_{2}}-\frac{\partial \varphi_{2}}{\partial x_{1}}+\lambda_{2}\left(\lambda_{2}-1\right) x_{2} \varphi_{1}} \\
& +R A\left(x_{1}, x_{2}, \varphi_{1}, \varphi_{2}, \frac{\partial \varphi_{1}}{\partial x_{1}}, \frac{\partial \varphi_{1}}{\partial x_{2}}, \frac{\partial \varphi_{2}}{\partial x_{1}}, R\right) \\
& +C\left(x_{1}, x_{2}, \varphi_{1}, \varphi_{2}, \frac{\partial \varphi_{1}}{\partial x_{1}}, \frac{\partial \varphi_{1}}{\partial x_{2}}, \frac{\partial \varphi_{2}}{\partial x_{1}}, R\right)=0, \\
& {\left[\lambda_{1}^{2} x_{1}^{2}+\lambda_{2}^{2} x_{2}^{2}+(1-Q)\right] \frac{\partial \varphi_{1}}{\partial x_{1}}+\frac{\partial \varphi_{2}}{\partial x_{2}}+\lambda_{1}\left(\lambda_{1}-1\right) x_{1} \varphi_{1}} \\
& +R \tilde{A}\left(x_{1}, x_{2}, \varphi_{1}, \varphi_{2}, \frac{\partial \varphi_{1}}{\partial x_{1}}, \frac{\partial \varphi_{1}}{\partial x_{2}}, \frac{\partial \varphi_{2}}{\partial x_{2}}, R\right) \\
& +\tilde{C}\left(x_{1}, x_{2}, \varphi_{1}, \varphi_{2}, \frac{\partial \varphi_{1}}{\partial x_{1}}, \frac{\partial \varphi_{1}}{\partial x_{2}}, \frac{\partial \varphi_{2}}{\partial x_{2}}, R\right)=0,
\end{aligned}
$$

where $A, \tilde{A}$ are smooth functions and $C, \tilde{C}$ are quadratic forms in $\varphi_{1}, \varphi_{2}, \frac{\partial \varphi_{1}}{\partial x_{1}}, \frac{\partial \varphi_{1}}{\partial x_{2}}$, $\frac{\partial \varphi_{2}}{\partial x_{1}}$ and $\frac{\partial \varphi_{2}}{\partial x_{2}}$ with smooth coefficients.

The equations (4.19) and (4.20) are the desired Cauchy-Riemann system. Setting $\varphi_{1}=\varphi_{2}=0$, we see that the initial disks $f^{0}$ are $J_{R}$-holomorphic up to a multiple of $R^{2}$. It is convenient to replace $\varphi_{1}$ by $\left[\lambda_{1}^{2} x_{1}^{2}+\lambda_{2}^{2} x_{2}^{2}+(1-Q)\right]^{-1} \varphi_{1}$ in (4.19) and (4.20). The resulting equations are

$$
\begin{aligned}
G_{2} & \equiv \frac{\partial \varphi_{1}}{\partial x_{2}}-\frac{\partial \varphi_{2}}{\partial x_{1}}+\alpha \varphi_{1}+R A+C=0, \\
G_{1} & \equiv \frac{\partial \varphi_{1}}{\partial x_{1}}+\frac{\partial \varphi_{2}}{\partial x_{2}}+\tilde{\alpha} \varphi_{1}+R \tilde{A}+\tilde{C}=0,
\end{aligned}
$$

where the functions $\alpha, \tilde{\alpha}, A, C, \tilde{C}$ and $\tilde{B}$ are defined in an obvious way. We consider the operator $G=\left(G_{1}, G_{2}\right)$,

$$
G: W^{k, \sigma}\left(\Omega \times \mathbb{R}^{2}, \partial \Omega \times \mathbb{R}\right) \rightarrow W^{k-1, \sigma}\left(\Omega \times \mathbb{R}^{2}\right),
$$


where $\mathbb{R}$ is identified with $\mathbb{R} \times\{0\}$. The linearization $L=G^{\prime}$ at the zero section reads

$$
L \varphi=L_{0} \varphi+R L(R) \varphi
$$

for $\varphi=\left(\varphi_{1}, \varphi_{2}\right)$, where $L(R)$ is a suitable first order operator with smooth parameter $R$ and

$$
L_{0} \varphi=\left(\frac{\partial \varphi_{1}}{\partial x_{1}}+\frac{\partial \varphi_{2}}{\partial x_{2}}+\tilde{\alpha} \varphi_{1}, \frac{\partial \varphi_{1}}{\partial x_{2}}-\frac{\partial \varphi_{2}}{\partial x_{1}}+\alpha \varphi_{1}\right) .
$$

Now we are ready to prove Theorem 1.

Proof of Theorem 1. Existence. The operator $L_{0}$ is a Cauchy-Riemann operator with respect to the conjugate complex structure on $\mathbb{R}^{2}$. On can convert it into the formulation of linear theory in the last section or [MS], but that's not necessary. It is clear that the Maslov class $\mu\left(\Omega \times \mathbb{R}^{2}, \partial \Omega \times \mathbb{R}\right)=0$. Hence $i$ ind $L_{0}=1$, and $L_{0}$ is surjective on account of Theorem 2.9. It follows that ind $L=1$ and $L$ is surjective, provided that $R$ is sufficiently small. Moreover, the following elliptic estimate holds (see $[\mathrm{MS}])$ :

$$
\|\varphi\|_{W^{k, \sigma}} \leq C\left(\|L \varphi\|_{W^{k-1, \sigma}}+\|\varphi\|_{L^{\sigma}}\right)
$$

where $\varphi \in W^{k, \sigma}\left(F \times \mathbb{R}^{2}, \partial F \times \mathbb{R}\right)$, assuming that $R$ is small enough. Here and in the sequel, $C$ denotes suitable positive constants independant of $R$. Now choose a small $R_{0}$ and set $L_{R_{0}}=L_{0}+R_{0} L\left(R_{0}\right)$. Let $K_{0}$ denote the kernel of $L_{R_{0}}$ and $W^{k, \sigma}\left(F \times \mathbb{R}^{2}, \partial F \times \mathbb{R}\right)=K_{0} \oplus K_{0}^{\perp}$ a decomposition. It follows from (4.23) that

$$
\|\varphi\|_{W^{k, \sigma}} \leq C\|L \varphi\|_{W^{k-1, \sigma}}
$$

for $\varphi \in K_{0}^{\perp}$, provided that $R_{0}$ and $R$ are small. One also sees that $\left.L\right|_{K_{0}^{\perp}}$ is surjective as long as $R_{0}$ and $R$ are small. We fix an $R_{0}>0$ such that all the above facts hold for $R_{0}$ and $0<R \leq R_{0}$.

Now we consider the operator $\tilde{G}=\left.G\right|_{K_{0}^{\perp}}$ with $R \leq R_{0}$. By the above facts $\tilde{G}^{\prime}(0)$ is an isomorphism with uniformly bounded inverse. By the implicit function theorem, $\tilde{G}$ is a diffeomorphism on a neighborhood $\mathcal{V}$ of the zero section such that $\tilde{G}(\mathcal{V})$ contains the ball $B_{\delta}(\tilde{G}(0))$ for a positive number $\delta$ independent of $R$. But $\|\tilde{G}(0)\| \leq C R$; hence $0 \in B_{\delta}(\tilde{G}(0))$ whenever $R$ is small enough. For such $R, \varphi_{R}=\left.\tilde{G}\right|_{\mathcal{V}} ^{-1}(0)$ is a solution of (4.21) and (4.22). We have

$$
\left\|\varphi_{R}\right\| \leq C R,
$$

as well as

$$
\left\|\frac{\partial}{\partial R} \varphi_{R}\right\| \leq C R .
$$

Let $f_{R}$ be the corresponding disks given by the formula (4.3). It follows from the above estimate that $f_{R}$ are embedded for small $R$. Hence image $\left(f_{R}\right)$ are $J_{R^{-}}$ holomorphic with boundary on the $\left(x_{1}, x_{2}\right)$-plane. By elliptic regularity, $f_{R}$ (or $\left.\varphi_{R}\right)$ are smooth. The family $\varphi_{R}$ is smooth as a family in a fixed Sobolev space. Applying the implicit function theorem in different Sobolev spaces, we deduce that $\varphi_{R}$ and hence $f_{R}$ are smooth families as smooth mappings.

Through the dilation of factor $R$ and the coordinate map, the $f_{R}$ yield $J$ holomorphic disks $F_{R}$ with boundary on $S \backslash\left\{p_{0}\right\}$ such that $F_{R} \rightarrow p_{0}$ as $R \rightarrow 0$. The parametrization induced by $f_{R}$ will be denoted by $\tilde{f}_{R}$. For a fixed $R_{1}$ we consider deformations of $F=F_{R_{1}}$ in the set-up of Section 2 or Section 3. It is easy 
to see that $\mu\left(V, V_{0}\right)=0$ here. The family $F_{R}$ for $R$ near $R_{1}$ can be represented by a family of sections $\varphi(R)$ of $V$ with boundary values in $V_{0}$. It follows that the derivative $\varphi^{\prime}\left(R_{1}\right)$ satisfies the equation

$$
L \varphi^{\prime}\left(R_{1}\right)=0 .
$$

Using the formula (4.3), one readily shows that $\varphi^{\prime}\left(R_{1}\right)$ is not identically zero. Since $\mu\left(V, V_{0}\right)=0$, we conclude from Theorem 2.9 that $\varphi^{\prime}\left(R_{1}\right)$ has no zero. Since $R_{1}$ is arbitary, we deduce from Theorem 2.9 that the family $\tilde{f}_{R}$ defines an immersion of $\Omega \times\left(0, R_{0}^{\prime}\right)$ for some $R_{0}^{\prime}>0$.

We claim that the union $N=\left(\bigcup_{0<R \leq R_{0}^{\prime}} F_{R}\right) \cup\left\{p_{0}\right\}$ is a $C^{1}$ embedded 3-ball, provided that $R_{0}^{\prime}$ has been chosen small enough. Consider the smooth map

$$
\begin{aligned}
& \Phi\left(x_{1}, x_{2}, x_{3}\right)=\left(x_{1}\left(1+\lambda_{1}\left(\varphi_{R}\right)_{1}\left(\frac{x_{1}}{R}\right)\right), \quad x_{2}\left(1+\lambda_{2}\left(\varphi_{R}\right)_{1}\left(\frac{x^{\prime}}{R}\right)\right),\right. \\
& \left.\left(Q\left(x^{\prime}\right)-R^{2}\right)\left(1+\left(\varphi_{R}\right)_{1}\left(\frac{x^{\prime}}{R}\right)\right), \quad R^{2}\left(\varphi_{R}\right)_{2}\left(\frac{x^{\prime}}{R}\right)\right),
\end{aligned}
$$

where $x^{\prime}=\left(x_{1}, x_{2}\right),\left(x_{1}, x_{2}, x_{3}\right) \in \Omega \backslash\{0\}$ with $\Omega=\left\{\left(x_{1}, x_{2}, x_{3}\right) \in \mathbb{R}^{3}: Q\left(x_{1}, x_{2}\right)\right.$ $\left.\leq\left(R_{0}^{\prime}\right)^{2}, 0 \geq x_{3} \geq Q\left(x_{1}, x_{2}\right)-\left(R_{0}^{\prime}\right)^{2}\right\}$, and $R=R\left(x_{1}, x_{2}, x_{3}\right)=\sqrt{Q\left(x_{1}, x_{2}\right)-x_{3}}$. It is easy to see that image $(\Phi)=\bigcup_{R} F_{R}$; indeed $\Omega \backslash\{0\}$ is foliated by an obvious family of parabloids and $\Phi$ sends them to the family $\left\{F_{R}\right\}$. By the estimate (4.23), $\Phi$ extends continuously to the origin with $\Phi(0)=0$. An easy computation combined with the estimates (4.23) and (4.24) then shows that the first order derivatives of $\Phi$ extend continuously to the origin with

$$
\frac{\partial}{\partial x_{1}} \Phi(0)=(1,0,0,0), \frac{\partial}{\partial x_{2}} \Phi(0)=(0,1,0,0), \frac{\partial}{\partial x_{3}}=(0,0,1,0) .
$$

It follows that $\Phi$ is an embedding near the origin. By Proposition 5.3 below, $N$ is $J$-flat.

\section{UNIQUENESS}

Consider two nonconstant $J$-holomorphic curves $f_{1}: \Sigma_{1} \rightarrow M$ and $f_{2}: \Sigma_{2} \rightarrow M$ such that $f_{1}\left(\partial \Sigma_{1}\right) \subset S^{*}$ and $f_{2}\left(\partial \Sigma_{2}\right) \subset S^{*}$ for a surface $S$. Assume $f_{1}\left(z_{1}\right)=$ $f_{2}\left(z_{2}\right)=p$ for some $z_{1} \in \partial \Sigma_{1}$ and $z_{2} \in \partial \Sigma_{2}$. For convenience, we shall say that the pointed maps $\left(f_{1}, z_{1}\right)$ and $\left(f_{2}, z_{2}\right)$ intersect at $p$.

Definition 5.1. We say that $\left(f_{1}, z_{1}\right)$ and $\left(f_{2}, z_{2}\right)$ intersect at $p$ from one side of $S$, provided that there is an orientable hypersurface $N$ containing a neighborhood of $p$ in $S$, such that $f_{1}(z)$ and $f_{2}\left(z^{\prime}\right)$ approach $N$ from the same side as $z$ approaches $\partial \Sigma_{1}$ around $z_{1}$ and $z^{\prime}$ approaches $\partial \Sigma_{2}$ around $z_{2}$. Moreover, image $\left(\left.d f_{1}\right|_{z_{1}}\right) \not \subset T_{p} N$ and image $\left(\left.d f_{2}\right|_{z_{2}}\right) \not \subset T_{p} N$ (in particular, neither $z_{1}$ nor $z_{2}$ is a branch point).

Now consider $\left(f_{1}, z_{1}\right), z_{1} \in \partial \Sigma_{1}$, and $\left(f_{2}, z_{2}\right), z_{2} \in \partial \Sigma_{2}$, as above, which intersect at $p$ from one side of $S$. Since the one side intersection is a local property, we can assume that $\Sigma_{1}=B^{+}=\stackrel{\circ}{B} \cap\{\operatorname{Im} z \geq 0\}$ as well as $\Sigma_{2}=B^{+}$. Let $f^{k}: \Sigma_{1} \rightarrow M$ be a sequence of $C^{1}$ maps such that $f^{k}\left(\partial \Sigma_{1}\right) \subset S^{*}$, each $f^{k}$ intersects $f$ only at isolated points (e.g. they are all $J$-holomorphic), and the $f^{k}$ converge to $f_{1}$ in $C^{1}$ as $k \rightarrow \infty$.

Lemma 5.2 (stability of one side intersections). Assume that the images of $f_{1}$ and $f_{2}$ near $z_{1}$ and $z_{2}$ do not overlap. For $k$ large enough, $f^{k}$ intersects $f_{2}$ near $p$. 
Proof. If $f_{1}$ intersects $f_{2}$ transversally at $p$, then the boundary of $f_{1}$ intersects the boundary of $f_{2}$ transversally (considered as curves on $S$ ). Hence the assertion of the lemma follows easily. Now we assume that the $f_{1}$ and $f_{2}$ intersect nontransversally at $p$. Restricting $f_{1}$ and $f_{2}$ suitably, we may assume that $f_{1}$ and $f_{2}$ are embedded. By restricting $f^{k}$ suitably, we can then assume that the $f^{k}$ are all embedded. Let $F_{i}=f_{i}\left(\Sigma_{i}\right), i=1,2$, and $F^{k}=f^{k}\left(\Sigma_{1}\right)$. We choose local coordinates $\left(x_{1}, x_{2}, x_{3}, x_{4}\right)$ around $p=f_{1}\left(z_{1}\right)=f_{2}\left(z_{2}\right)$ with the following properties: $p$ corresponds to the origin, $F_{2}$ is represented by the closed upper half $\left(x_{1}, x_{2}\right)$-plane, $S$ is represented by the $\left(x_{1}, x_{3}\right)$-plane, $J \frac{\partial}{\partial x_{1}}=\frac{\partial}{\partial x_{2}}$ and $J \frac{\partial}{\partial x_{3}}=\frac{\partial}{\partial x_{4}}$. Restricting $F_{1}$ and $F_{2}$ suitably, we can represent $F_{1}$ as the graph of a function $w=h(z)$ over a closed disk type domain $\Omega_{0}$ in the closed upper half $\left(x_{1}, x_{2}\right)$-plane such that $I_{0}=\partial \Omega_{0} \cap\left\{x_{2}=0\right\}$ is an interval containing the origin, where $z=x_{1}+\sqrt{-1} x_{2}$ and $w=x_{3}+\sqrt{-1} x_{4}$. This follows from the nontransversal and one side assumptions. For $k$ large we can then represent (a portion of) $F^{k}$ as the graph of a function $w=h_{k}(z)$ over $\Omega_{0}$ such that the $h_{k}$ converge to $h$ in $C^{1}$. Notice that $\operatorname{Im}\left(\left.h\right|_{I_{0}}\right) \equiv 0$ and $\operatorname{Im}\left(\left.h_{k}\right|_{I_{0}}\right) \equiv 0$, because $f\left(\partial \Sigma_{1}\right) \subset S$ and $f^{k}\left(\partial \Sigma_{1}\right) \subset S$. Let $\Omega_{0}^{-}=\left\{z: \bar{z} \in \Omega_{0}\right\}$ and $\tilde{\Omega}_{0}=\Omega_{0} \cup \Omega_{0}^{-}$. We extend $h$ to a function $\tilde{h}$ on $\tilde{\Omega}_{0}$ by requiring $\tilde{h}(z)=\tilde{\tilde{h}}(\bar{z})$. Because $\operatorname{Im}\left(\left.h\right|_{I_{0}}\right) \equiv 0$, this is a continuous extension. We extend $h_{k}$ to $\tilde{h}_{k}$ in the same fashion.

By the arguments for the asymptotic behavior of pseudo holomorphic curves in [Y1], we have

$$
h(z)=\alpha z^{l}+O\left(|z|^{l+1}\right)
$$

for an integer $l \geq 2$ and a nonzero complex number $\alpha$. (The integer $l$ is at least 2 , because $F_{1}$ and $F_{2}$ are tangent at $p$.) Here we have used the non-overlapping assumption to rule out the possibility that $h$ is identically zero. Because $\operatorname{Im}\left(\left.h\right|_{I_{0}}\right) \equiv$ $0, \alpha$ is real, whence

$$
\tilde{h}(z)=\alpha z^{l}+O\left(|z|^{l+1}\right) .
$$

Consequently,

$$
\tilde{h}_{k}(z)=\alpha z^{l}+O\left(|z|^{l+1}\right)+\varepsilon_{k}(z),
$$

where $\varepsilon_{k}(z) \rightarrow 0$ as $k \rightarrow \infty$. By the mapping degree theory, $\tilde{h}_{k}$ has $l$ zeros near the origin whenever $k$ is large enough. Since $\tilde{h}_{k}(z)=\overline{\tilde{h}_{k}(\bar{z})}, h_{k}$ has at least $\left[\frac{l}{2}\right]$ zeros near the origin. This means that $f^{k}$ intersects $f_{2}$ near $p$.

Remark. A (homotopy invariant) quantitative formulation of this result will be given in Section 7.

Next we consider an orientable hypersurface $N$ in $M$. Let $\xi$ be the field of complex planes on $N$. Following [E1], we say that $N$ is $J$-convex if $d \alpha(J v, v)>0$ (or $d \alpha(J v, v)<0$ ) for all nonzero vectors $v \in \xi_{p}, p \in N$, where $\alpha$ is a 1-form such that $\xi=\{v \in T N: \alpha(v)=0\}$. We say that $N$ is $J$-flat, if $d \alpha(J v, v)=0$ for all $v \in \xi$. A simple computation along with Frobenius' theorem leads to the following fact.

Proposition 5.3. $N$ is $J$-flat if and only if $N$ is foliated by embedded J-holomorphic curves.

Now we assume that $N$ is $J$-convex. For a function $h$ in a neighborhood of $N$ such that $d h \neq 0$ everwhere and $N=\{h=0\}$, we can set $\alpha=J d h \equiv d h \circ J$. We choose an $h$ such that $d(J d h)(J v, v)>0$ for all $v \in \xi$. The side of $N$ where $h \leq 0$ 
will be called the convex side of $N$. The other side is called the concave side of $N$. We call $h$ a level function of $N$. Moreover, if $N=\partial M$ and the convex side of $N$ is the inside, then we say that $M$ has $J$-convex boundary $\partial M$.

Let $f: \Sigma \rightarrow M$ be a $J$-holomorphic curve. We have

Lemma 5.4. If $f\left(z_{0}\right) \in N$ and $f$ is tangent to $N$ at $z_{0}$, then $\Delta(h \circ f) \geq 0$ in a neighborhood of $z_{0}$, where $\Delta$ is the Laplacian of some conformal coordinates around $z_{0}$. Moreover, $\Delta(h \circ f)(z)>0$ for $z$ in this neighborhood, provided that $d f(z) \neq 0$.

Proof. It follows from the local expansion of $f$ near its branch points in [Y1] that there is a continuous field of tangent planes $\xi_{0}$ along $f$ such that $\xi_{0}(z)$ is the image of $d f(z)$ whenever $z$ is not a branch point. By the assumption, $\xi_{0}\left(z_{0}\right)=\xi_{f\left(z_{0}\right)}$. We set $\tilde{\xi}=\{\alpha=0\}$, which is an extension of $\xi$ to a neighborhood of $N$. Let $P$ denote the orthogonal projection to $\tilde{\xi}$. Then there are a neighborhood $U$ of $N$ and a positive number $\delta$ such that

$$
d(J d h)(J v, v)>0
$$

for $v \in T_{p} M, p \in U$ with $v \neq 0$ and $\|P v\| \geq(1-\delta)\|v\|$. Now we have, for conformal coordinates $x_{1}, x_{2}$ around $z_{0}$,

$$
\Delta(h \circ f)=d(j d(h \circ f))\left(\frac{\partial}{\partial x_{2}}, \frac{\partial}{\partial x_{1}}\right)=d(J d h)\left(J \frac{\partial f}{\partial x_{1}}, \frac{\partial f}{\partial x_{1}}\right),
$$

where $j$ denotes the almost complex structure of $\Sigma$. Since $\xi_{0}\left(z_{0}\right)=\xi_{f\left(z_{0}\right)}$, there is a neighborhood of $z_{0}$ in which $\left\|P \frac{\partial f}{\partial x_{1}}\right\| \geq(1-\delta)\left\|\frac{\partial f}{\partial x_{1}}\right\|$. Then the conclusions of the lemma follow from (5.1) and (5.2).

Lemma 5.5. Assume that $f$ is nonconstant and $h \circ f \leq 0$ in a neighborhood of some $z_{0} \in \Sigma$. If $z_{0} \in \stackrel{\circ}{\Sigma}$, then $h \circ f\left(z_{0}\right)<0$ and hence $f\left(z_{0}\right) \notin N$. If $z_{0} \in \partial \Sigma$ and $f\left(z_{0}\right) \in N$, then $f$ is immersed at $z_{0}$ and $f$ is not tangent to $N$ at $z_{0}$.

Proof. The first statement follows from Lemma 5.4 and the maximum principle. In the second case, it follows from Lemma 5.4 and the Hopf boundary point lemma that $f$ is not tangent to $N$ at $z_{0}$ (i.e. $\left.\left.\xi_{0}\left(z_{0}\right)\right) \not \subset T_{f\left(z_{0}\right)} N\right)$. If $f$ were not immersed at $z_{0}$, then the local expansion given in [Y1] would imply that $h \circ f \leq 0$ could not hold in any neighborhood of $z_{0}$.

Now we proceed to prove the uniqueness part of Theorem 1. Consider the disks $F_{R}, 0<R \leq R_{0}^{\prime}$, constructed in section 4 . We observe that every $F_{R}$ lies in the region $x_{3} \leq 0$, provided that $R_{0}^{\prime}$ has been chosen small enough. This follows easily from the construction.

Next set $h_{0}=x_{3}, N_{0}=\left\{x_{3}=0\right\}$, and let $\xi$ be the field of complex planes on $N_{0}$. Then $\xi_{p_{0}}=\operatorname{span}\left(\frac{\partial}{\partial x_{1}}, \frac{\partial}{\partial x_{2}}\right)$. We compute at $p_{0}$ :

$$
\begin{aligned}
d\left(J d h_{0}\right)\left(J \frac{\partial}{\partial x_{1}}, \frac{\partial}{\partial x_{1}}\right) & =d\left(J d h_{0}\right)\left(\frac{\partial}{\partial x_{2}}, \frac{\partial}{\partial x_{1}}\right) \\
& =\frac{\partial}{\partial x_{2}} J d h_{0}\left(\frac{\partial}{\partial x_{1}}\right)-\frac{\partial}{\partial x_{1}} J d h_{0}\left(\frac{\partial}{\partial x_{2}}\right) \\
& =\frac{\partial}{\partial x_{2}} d h_{0}\left(\sum_{j=1}^{4} J_{1 j} \frac{\partial}{\partial x_{j}}\right)-\frac{\partial}{\partial x_{1}} d h_{0}\left(\sum_{j=1}^{4} J_{2 j} \frac{\partial}{\partial x_{j}}\right) \\
& =\frac{\partial}{\partial x_{2}} J_{13}-\frac{\partial}{\partial x_{1}} J_{23}=b-c=\lambda_{1}+\lambda_{2}>0 .
\end{aligned}
$$


Then also

$$
d\left(J d h_{0}\right)\left(J \frac{\partial}{\partial x_{2}}, \frac{\partial}{\partial x_{2}}\right)=d\left(J d h_{0}\right)\left(-\frac{\partial}{\partial x_{1}}, \frac{\partial}{\partial x_{2}}\right)=\lambda_{1}+\lambda_{2} .
$$

On the other hand,

$$
\begin{aligned}
& d\left(J d h_{0}\right)\left(J \frac{\partial}{\partial x_{2}}, \frac{\partial}{\partial x_{1}}\right)=-d\left(J d h_{0}\right)\left(\frac{\partial}{\partial x_{1}}, \frac{\partial}{\partial x_{1}}\right)=0, \\
& d\left(J d h_{0}\right)\left(J \frac{\partial}{\partial x_{1}}, \frac{\partial}{\partial x_{2}}\right)=d\left(J d h_{0}\right)\left(\frac{\partial}{\partial x_{2}}, \frac{\partial}{\partial x_{2}}\right)=0 .
\end{aligned}
$$

It follows that $d\left(J d h_{0}\right)(J v, v)>0$ for all nonzero $v \in \xi_{p_{o}}$. By continuity,

$$
d\left(J d h_{0}\right)(J v, v)>0
$$

for all nonzero $v \in \xi_{p}$ whenever $p \in N_{0} \cap U_{1}$ for some neighborhood $U_{1}$ of $p_{0}$. Moreover, we can choose $U_{1}$ such that $d\left(J d h_{0}\right)(J v, v)>0$ for all nonzero $v \in \xi_{p}^{t}, p \in$ $N_{t} \cap U_{1}$ and $t \in(-\varepsilon, \varepsilon)$, where $\varepsilon$ is a positive number, $N_{t}=\left\{x_{3}=t\right\}$ and $\xi^{t}$ denotes the field of complex planes on $N_{t}$. Thus we have a family of $J$-convex hypersurfaces $N_{t} \cap U_{1}$ along with level functions $h_{t}=h_{0}-t$. We set $S^{\prime}=\overline{\bigcup_{0<R \leq R_{1}} \partial F_{R}}$ for some $R_{1}$ such that $S^{\prime} \subset U_{1}$.

Proposition 5.6 (One side global uniqueness). Let $f: \Sigma \rightarrow M$ be a nonconstant J-holomorphic curve such that $f(I) \subset S^{\prime}$ and $f(\partial I) \subset \partial S^{\prime}$, where $\Sigma$ is a compact Riemann surface with boundary and $I$ is a nonempty connected subset of $\partial \Sigma$ ( $\partial I$ may be empty). Assume that $f(z)$ approaches $S^{\prime}$ in the region $x_{3} \leq 0$ as $z \rightarrow \stackrel{\circ}{I}$ and $p_{0} \notin$ image $(f)$. Then $f$ is a holomorphic covering onto $F_{R}$ for some $R$.

Proof. For $R$ small enough, $F_{R}$ is disjoint from $f$. On the other hand, $f$ must intersect some $F_{R}$ along the boundary. Hence we can find an $R$ such that $f$ intersects $F_{R}$ and is disjoint from $F_{R^{\prime}}$ for $R^{\prime}<R$. We claim $f$ is a holomorphic covering onto $F_{R}$, which is equivalent to saying that $\operatorname{image}(f)=F_{R}$. Assume that this is not true. If $f$ intersects $F_{R}$ at an interior point, then it must intersect $F_{R^{\prime}}$ at an interior point for $R^{\prime}$ close to $R$. Indeed, this is clear if the intersection is transversal. If it is not transversal, we can argue as in the proof of Lemma 5.2. Hence there is no interior intersection between $f$ and $F_{R}$. It follows that $f$ must intersect $F_{R}$ at the boundary. Applying Lemma 5.5 and Lemma 5.2, we then arrive at a contradiction.

Remark. One can remove the assumption that $p_{0} \notin i m a g e(f)$ by the following fact, which is easy to verify: the $J$-flat hypersurface $\left(\bigcup_{R} F_{R}\right) \cup\left\{p_{0}\right\}$ has arbitarily small neighborhoods with $J$-convex boundary. This was pointed out to me by Eliashberg. This remark also applies to Proposition 6.2 and Theorem 8.8.

To proceed, we choose a neighborhood $\tilde{U}$ of $p_{0}$ such that $\tilde{U} \subset U_{1}$ and $F_{R_{1}}$ is disjoint from $\tilde{U}$.

Proposition 5.7 (strong local uniqueness). Let $f: \Sigma \rightarrow M$ be a compact J-holomorphic curve with image contained in $\tilde{U}$, such that $f(\partial \Sigma) \subset S$ in the case $\partial \Sigma \neq \emptyset$. If $f$ has empty boundary, then $f$ must be constant. If $f$ is nonconstant, then $f$ is a holomorphic covering onto $F_{R}$ for some $R$.

Proof. There is a point $p_{1}$ in the image of $f$ where $h$ achieves its maximal value $t_{1}$. Applying Lemma 5.5 with $N=N_{t_{1}}$ and $h=h_{t_{1}}$, conclude that $p_{1}$ must be a boundary point, provided that $f$ is nonconstant. Now assume that $f$ is nonconstant. 
We conclude that the image of $f$ is contained in the region $x_{3} \leq 0$. Arguing as in the proof of Proposition 5.6, we deduce that image $(f)=F_{R}$ for some $R$.

Proof of Theorem 1. Uniqueness. The uniqueness assertion follows from Proposition 5.7.

\section{Compactness and Continuation}

In this section we prove Theorem 2. First we reformulate Gromov's compactness theorem for pseudoholomorphic curves as proved in [Y1] (and some implications of the proof in [Y1]) in a convenient way. We only need the case of disks here. The general case can be formulated in a similar way.

Gromov's Compactness Theorem. Assume that $(M, J)$ has bounded geometry, i.e. there is an Hermitian metric $g$ with uniformily bounded sectional curvatures and positive injectivity radius such that $J$ has uniformly bounded covariant derivative. Let $S_{1} \subset M$ be a compact, totally real surface (i.e. $S_{1}$ consists of real points) and $f_{k}$ a sequence of J-holomorphic disks with boundary on $S_{1}$ and with uniformly bounded area (measured in $g$ ). Then there are a subsequence of $f_{k}$, which we still denote by $f_{k}$, and a finite set $\Gamma \subset B$, such that $f_{k}$ converges smoothly on $B \backslash \Gamma$ to a smooth J-holomorphic disk $f_{\infty}^{0}$ with boundary on $S_{1}$. Blow-up limits $f_{\infty}^{1}, \ldots, f_{\infty}^{m}$ arise at points of $\Gamma$, which are either non-constant J-holomorphic spheres or disks with boundary on $S_{1}$. (J-holomorphic spheres arise at points in $\Gamma \cap \stackrel{\circ}{B}$, while at least one disk arises at each point in $\Gamma \cap \partial B$.) The curves $f_{\infty}^{0}, \ldots, f_{\infty}^{m}$ constitute a $J$-cusp-curve $f$ (in particular the union of their images is connected) such that $f_{k}$ converge to $f$ in the Gromov topology for cusp-curves. Consequently, we have:

1. $\operatorname{area}\left(f_{k}\right)$ converges to area $(f)=\sum_{i=0}^{m} \operatorname{area}\left(f_{\infty}^{i}\right)$.

2. image $\left(f_{k}\right)$ converges to image $(f)=\bigcup_{i=0}^{m}$ image $\left(f_{\infty}^{i}\right)$ in the Hausdorff distance.

3. For large $k$, the $f_{k}$ are mutually homotopic with boundary confined on $S$. In particular the following equation for the Maslov classes holds:

$$
\mu\left(f_{k}\right)=\mu(f)=\mu\left(f_{\infty}^{0}\right)+\ldots+\mu\left(f_{\infty}^{m}\right) .
$$

4. For large $k$, the homology class $\left[\left.f_{k}\right|_{\partial B}\right]$ in $S_{1}$ is equal to the sum of the homology classes $\left[\left.f_{\infty}^{i}\right|_{\partial B}\right]$, where $f_{\infty}^{i}$ runs over all disks in $f$.

In $[\mathrm{Y} 1]$ one can find more detailed descriptions of the convergence of $f_{k}$ to $f$. We shall need the following fact.

Corollary. Let $z_{0} \in \Gamma \cap \partial B$. Then there are round disks $A_{k}=B_{r_{k}}\left(z_{k}\right)$ of radius $r_{k} \rightarrow 0$ centered at $z_{k} \rightarrow z_{0}$ with $z_{k} \in \partial B \backslash \Gamma$ along with conformal injections $\psi_{k}: A_{k} \rightarrow H \equiv\{z \in \mathbb{C}: \operatorname{Im} z \geq 0\}$ such that $\psi_{k}\left(\partial A_{k} \cap \partial B\right) \subset \partial H, \psi_{k}\left(A_{k}\right) \rightarrow H$, and $f_{k} \circ\left(\psi_{k}\right)^{-1}$ converge smoothly away from finitely many points to one component of $f$, say $f_{\infty}^{1}$. Moreover, the $f_{k}\left(\partial A_{k} \backslash \partial B\right)$ converge to a cusp point $p_{0}$ of $f$ at which $f_{\infty}^{0}$ and $f_{\infty}^{1}$ intersect.

From now on we assume that $M$ has $J$-convex boundary $\partial M$. Consider a compact and orientable surface $S \subset \partial M$ with finitely many complex points. Let $\eta$ denote the intersection of $\xi$ with the tangent planes of $S^{*}$, where $\xi$ is the field of complex planes on $\partial M$. In [E1], $\eta$ is called the characteristic direction. Since $S$ is orientable, we can represent $\eta$ by a nowhere vanishing smooth vector field, which will also be denoted by $\eta$. 
Proposition 6.1 (Smooth Compactness). Assume that $(M, J)$ has bounded geometry and contains no nonconstant J-holomorphic sphere. Let $\tilde{f}_{k}$ be a sequence of embedded (parametrized) $J$-holomorphic disks in $M$ with boundary on $S$, such that $\tilde{f}_{k}$ has uniformly bounded area and the boundary of $\tilde{f}_{k}$ has uniformly positive distance from the set of complex points of $S$. Moreover, assume that for each $k$, the homology class $\left[\left.f_{k}\right|_{\partial B}\right]$ in $S$ is trivial. Then a subsequence of $\tilde{f}_{k} \circ \varphi_{k}$ converges smoothly to an embedded J-holomorphic disk $f$ with boundary on $S$, where $\varphi_{k}$ is a suitable sequence of conformal automorphisms of the unit disk.

Proof. For suitable conformal automorphisms $\varphi_{k}$, we have $\left\|d f_{k}(0)\right\|=1$, where $f_{k}=\tilde{f}_{k} \circ \varphi_{k}$. We apply Gromov's compactness theorem to the sequence $f_{k}$. Since there exists no nonconstant $J$-holomorphic sphere, the singular set $\Gamma$ is contained in $\partial B$. In particular, the $f_{k}$ converge smoothly in the interior. Then it follows from the condition $\left\|d f_{k}(0)\right\|=1$ that the limit $f_{\infty}^{0}$ is nonconstant. We claim that $\Gamma$ is empty. Assume that there is one $z_{0} \in \Gamma$. Consider the $A_{k}, \psi_{k}, f_{\infty}^{1}$ and $p_{0}$ as described in the above corollary. It follows from Lemma 5.4 that $f_{\infty}^{0}$ and $f_{\infty}^{1}$ are immersed along the boundary. One readily sees that $p_{0} \in S^{*}$. Let $z_{1}, z_{2} \in \partial B$ be such that $f_{\infty}^{0}\left(z_{1}\right)=f_{\infty}^{1}\left(z_{2}\right)=p_{0}$. By the assumption on the homology class, $\left.f_{k}\right|_{\partial B}$ bounds a disk in $S$. Using this fact and the fact that $f_{\infty}^{0}, f_{\infty}^{1}$ are immersed along the boundary, it is easy to see that there are $\operatorname{arcs} I_{1}, I_{2} \subset \partial B$ such that $z_{1} \in \stackrel{\circ}{I}_{1}, z_{2} \in \stackrel{\circ}{I}_{2},\left.f_{\infty}^{0}\right|_{I_{1}}$ and $\left.f_{\infty}^{1}\right|_{I_{2}}$ are embedded, and $f_{\infty}^{0}\left(I_{1}\right)$ lies on one side of $f_{\infty}^{1}\left(I_{2}\right)$. Consequently, $f_{\infty}^{0}\left(I_{1}\right)$ and $f_{\infty}^{1}\left(I_{2}\right)$ are tangent to each other at $p_{0}$.

Choose a continuous unit tangent vector field $X_{0}$ on $S^{*}$ such that $X_{0}$ is orthogonal to $\eta$ everywhere. Let $\tau$ be a continuous unit tangent vector field of $\partial B$. By the convergence of $f_{k}$ to $f_{\infty}^{0}$ and that of $f_{k} \circ\left(\psi_{k}\right)^{-1}$ to $f_{\infty}^{1}$, the bounding property of $\left.f_{k}\right|_{\partial B}$ and the local structure of $f_{\infty}^{0}$ and $f_{\infty}^{1}$ near $z_{1}$ and $z_{2}$, we can find for each large $k$ two points $w_{k} \in \partial B \backslash \Gamma$ and $\tilde{w}_{k} \in \partial B \cap A_{k}$, such that $\tilde{w}_{k}$ are near one end of $\partial A_{k} \cap \partial B$ and $w_{k}$ near the other, $f_{k}\left(w_{k}\right) \rightarrow p_{0}, f_{k}\left(\tilde{w}_{k}\right) \rightarrow p_{0}$, and

$$
\lim _{k \rightarrow \infty} Y_{k}\left(w_{k}\right)=v, \lim _{k \rightarrow \infty} Y_{k}\left(\tilde{w}_{k}\right)=-v,
$$

where $Y_{k}=\frac{\partial f_{k}}{\partial \tau}\left(\left|\frac{\partial f_{k}}{\partial \tau}\right|^{-1}\right)$ and $v$ is a unit tangent vector of $f_{\infty}^{0}\left(L_{1}\right)$ at $p_{0}$. Observe that $v \cdot X_{0}\left(p_{0}\right) \neq 0$, as otherwise $v \in \eta$ and hence $f_{\infty}^{0}$ would be tangent to $N$ at $p_{0}$, contradicting Lemma 5.4. Hence, for large $k$, the products $\frac{\partial f_{k}}{\partial \tau}\left(w_{k}\right) \cdot X_{0}\left(f_{k}\left(w_{k}\right)\right)$ and $\frac{\partial f_{k}}{\partial \tau}\left(\tilde{w}_{k}\right) \cdot X_{0}\left(f_{k}\left(\tilde{w}_{k}\right)\right)$ have opposite signs. By the mean value theorem, there is some $\bar{w}_{k} \in \partial B$ such that $\frac{\partial f_{k}}{\partial \tau}\left(\bar{w}_{k}\right) \cdot X_{0}\left(f_{k}\left(\bar{w}_{k}\right)\right)=0$. But this is impossible, because by Lemma 5.5, $\left.f_{k}\right|_{\partial B}$ is transversal to the characterstic direction $\eta$. We conclude that $\Gamma$ is empty.

We would like to present a somewhat different argument under the assumption that the homology classes $\left[\left.f_{k}\right|_{\partial B}\right]$ in $S^{*}$ (instead of in $S$ ) are indecomposable (this is the case in the latter applications of this proposition). By Gromov's compactness theorem, we have

$$
\left[\left.f_{k}\right|_{\partial B}\right]=\left[\left.f_{\infty}^{0}\right|_{\partial B}\right]+\ldots+\left[\left.f_{\infty}^{m}\right|_{\partial B}\right]
$$

for large $k$. By the indecomposabilty assumption, all the terms but one on the right hand sides are zero. Say $\left[\left.f_{\infty}^{1}\right|_{\partial B}\right]=0$. As before, $f_{\infty}^{1}$ is an immersion along the boundary. From the construction of the limit $J$-cusp-curve $f$ in [Y1] (basically the above corollary), it is easy to see that the only possible self-intersections of $\left.f_{\infty}^{1}\right|_{\partial B}$ are one-sided, i.e. one embedded portion meets another from one side (this 
includes the case of covering). Applying a factorization if necessary, we can assume that $f_{\infty}^{1}$ is not a multiple covering. Because the homology class $\left[\left.f_{\infty}^{1}\right|_{\partial B}\right]$ equals zero, $\left.f_{\infty}^{1}\right|_{\partial B}$ bounds a domain in $S^{*}$ which is either a disk or a union of several disks. Using the transversality with $\eta$ as above, one readily derives that $\left.f_{\infty}^{1}\right|_{\partial B}$ has no self-intersection, and hence it bounds a single disk $\Omega$ in $S^{*}$. Because $\eta$ is transversal to $\partial \Omega$, it must have a zero inside $\Omega$. This is impossible, because $\eta$ has no zeros.

We have shown that the $f_{k}$ converge smoothly on $B$ to $f_{\infty}^{0}$. Applying the intersection arguments in the proof of Proposition 5.6, we conclude that $f_{\infty}^{0}$ is an embedding along the boundary up to a covering map from the unit circle onto itself. But the covering map must be a diffeomorphism because the $f_{k}$ are embeddings and their boundaries are on an orientable surface. Hence $f_{\infty}^{0}$ is an embedding along the boundary. By the adjunction formula for $J$-holomorphic curves with boundary in the next section, we immediately conclude that $f_{\infty}^{0}$ is an embedding. It is also possible to use the adjunction formula for closed $J$-holomorphic curves in [M1] in the following way. Embed $M$ into a suitable larger manifold $M_{1}$ and glue a suitable embedded disk to each $f_{k}$ in such a way that the resulting sphere $h_{k}$ is a smooth embedding. Moreover, $h_{k}$ converges smoothly to some $h$ as $k$ goes to infinity in such a way that the only branch points and self-intersections of $h$ are in the interior of $f$. Then we construct almost complex structures $J_{k}$ on $M_{1}$ so that they coincide with $J$ on $M$ and $h_{k}$ is $J_{k}$-holomorphic. Moreover, $J_{k}$ converges smoothly as $k$ goes to infinity. Now we can apply the adjunction formula in [M1].

Note that if $f$ has no interior branch points, then the intersection argument cited above implies that $f$ is an embedding. Hence another possible (local) argument for proving the embedding property is to rule out interior branch points first. A local version of the adjunction formula in Section 7 provides such an argument. On the other hand, one can also use the results in [M2] and [MW] on knot types of singularities, at least under the assumption that the normal Maslov class of $\left[f_{k}\right]$ is zero for large $k$ (this is the case in the proof of Theorem 2 below). We omit the simple argument.

Now we are ready to prove Theorem 2 .

Proof of Theorem 2. First we notice that Definition 1.1 extends straightforwardly to manifolds with boundary. To apply Theorem 1 , we extend $(M, J)$ slightly to obtain a manifold $(\tilde{M}, J)$ such that $M$ lies in the interior of $\tilde{M}$. Let $\mathcal{G}_{0}=\left\{F_{t}\right\}_{0<t<1}$ be the local filling family near $p_{1}$ provided by Theorem 1 . We claim that this family $\left\{F_{t}\right\}$ is entirely contained in $M$. Indeed, the hypersurfaces $\partial_{\varepsilon} M=\{p \in \tilde{M}: h(p)=$ $\varepsilon\}$ in $\tilde{M}$ are J-convex for small $\varepsilon>0$, where $h$ is a level function of $\partial M$. Employing these hypersurfaces and Lemma 5.4, one deduces that all $F_{t}$ are contained in $M$. Similarly, the local filling family $\mathcal{G}_{1}$ near $p_{2}$ is also contained in $M$.

Choose a maximal integral curve $\mathcal{L}_{0}$ of the line field $\eta$ on $S \backslash\left\{p_{1}, p_{2}\right\}$ along with an (embedded) parametrization $\beta_{0}$ on the interval $(0,1)$ such that $\beta_{0}(t) \rightarrow p_{1}$ as $t \rightarrow 0$. Such a maximal integral curve exists because the family $\partial F_{t}$ is transversal to $\eta$ (Lemma 5.4). Also by this transversality, each $F_{t}$ intersects $\mathcal{L}_{0}$ at a unique point. We reparametrize the family $\left\{F_{t}\right\}_{0<t<1}$ to obtain $\left\{F_{t}\right\}_{0<t<\varepsilon}$ for some $\varepsilon>0$ such that $\beta_{0}(t) \in F_{t}$. Choose two more maximal integral curves $\mathcal{L}_{1}$ and $\mathcal{L}_{2}$, and set $\beta_{1}(t)=F_{t} \cap \mathcal{L}_{1}, \beta_{2}(t)=F_{t} \cap \mathcal{L}_{2}$. Fix three points $z_{0}, z_{1}$ and $z_{2}$ on $\partial B$, and let 
$f_{t}: B \rightarrow M$ be the $J$-holomorphic embedding with $f_{t}(B)=F_{t}, f_{t}\left(z_{i}\right)=\beta_{i}(t), i=$ $0,1,2$. Then the family $\mathcal{F}_{0}=\left\{f_{t}\right\}_{0<t<\varepsilon}$ yields a smooth embedding of $B \times(0, \varepsilon)$.

An extension of $\mathcal{F}_{0}$ is defined to be a smooth 1-parameter family $\mathcal{F}=\left\{f_{t}\right\}_{0<t<T}$, $\varepsilon \leq T<1$ of $J$-holomorphic disks with boundary on $S^{*}$ such that it yields a smooth embedding of $B \times(0, T), f_{t}$ is the same $f_{t}$ in $\mathcal{F}_{0}$ for $t<\varepsilon$, and $f_{t}\left(z_{i}\right)=\beta_{i}(t), i=$ $0,1,2$. Let $\mathcal{F}^{*}=\left\{f_{t}\right\}_{0<t<T^{*}}$ be a maximal extension. Set $F_{t}=\operatorname{image}\left(f_{t}\right)$. There are two cases to consider.

Case 1. There is some $t_{0}<T^{*}$ such that $F_{t_{0}}$ intersects some $F \in \mathcal{G}_{1}$.

Applying the intersection arguments in the proof of Proposition 5.6, one easily deduces that $F_{t_{0}}=F$. We apply the deformation theory in Section 2 to $F$. By homotopy invariance of the Maslov class, $\mu_{o}(F)$ is zero. Hence Proposition 2.5 and Theorem 2.9 imply that there is a unique 1-parameter family of $J$-holomorphic disks with boundary on $S$ around $F$ (modulo reparametrizations). We derive that the union $\mathcal{G}$ of the families $\left\{F_{t}, 0<t<T^{*}\right\}$ and $\mathcal{G}_{1}$ is a smooth family. Using the transversality it is then easy to show that the integral curves $\beta_{i}, i=0,1,2$, all run to $p_{2}$ as $t$ goes to 1 . Using these curves, we can parametrize the disks in $\mathcal{G}$ to obtain an extension of $\mathcal{F}_{0}$ which contains $\mathcal{F}^{*}$. Since the latter is a maximal extension, it is equal to the former. It follows that $\mathcal{F}^{*}$ is a desired family. Moreover, it is easy to see that $T^{*}=1$.

Case 2. $\bigcup_{0<t<T^{*}} F_{t}$ is disjoint from every disk in $\mathcal{G}_{1}$.

The taming condition implies that the $F_{t}$ have uniformly bounded area. Consider a sequence $t_{k}<T^{*}, t_{k} \rightarrow T^{*}$. The assumtion of the case implies that their boundaries have uniformly positive distance from $p_{2}$. By Proposition 6.1, a subsequence of $F_{t_{k}}$ converges smoothly to an embedded $J$-holomorphic disk $F$ with boundary on $S^{*}$. We apply the deformation theory in Section 2 to $F$ as before. Again we have $\mu_{o}(F)=0$, and hence there is a 1-parameter family of embedded $J$-holomorphic disks $\hat{F}_{t}$ with boundary on $S^{*},-\varepsilon^{\prime}<t<\varepsilon^{\prime}$, such that $\hat{F}_{0}=F$ and it yields an embedding of $F \times\left(-\varepsilon^{\prime}, \varepsilon^{\prime}\right)$. Applying Proposition 2.5, we deduce that the $F_{t_{k}}$ are members of this family for $k$ large. Then it is easy to see that the union $\mathcal{G}$ of this family with $\left\{F_{t}, 0<t<T^{*}\right\}$ is a smooth family which covers a neighborhood of $\partial F$ in $S$. It follows that the integral curves $\beta_{i}, i=0,1,2$, meet $\partial F$ transversally. Using these curves, we can parametrize $\mathcal{G}$ to obtain an extension of $\mathcal{F}_{0}$ which contains $\mathcal{F}^{*}$ properly, contradicting the maximality of $\mathcal{F}^{*}$. We conclude that Case 2 cannot happen.

The uniqueness of the family $\mathcal{F}^{*}$ or rather the family $\left\{F_{t}, 0<t<1\right\}$ follows from Proposition 6.2 below.

We note the following consequence of Theorem 2:

Corollary. The characteristic direction $\eta$ has no limit cycle. Indeed all its integral curves run from $p_{1}$ to $p_{2}$.

Proposition 6.2 (global uniqueness). Let $\mathcal{F}^{*}=\left\{f_{t}\right\}_{0<t<1}$ be the family constructed in the above proof. Let $f$ be a nonconstant J-holomorphic curve with nonempty boundary on $S$. Assume $\left\{p_{1}, p_{2}\right\} \not \subset$ image $(f)$. Then $f$ is a holomorphic covering onto image $\left(f_{t}\right)$ for some $t$.

This is similar to Proposition 5.6. We omit the proof. 


\section{The Adjunction Formula}

Consider $(M, J)$ and an oriented surface $S \subset \partial M$. Let $f: \Sigma \rightarrow M$ be a continuous map from a Riemann surface $\Sigma$ with boundary such that $f(\partial \Sigma) \subset S$. Such an $f$ will be called a parametrized surface with boundary on $S$. An admissible parametrized surface is either a parametrized surface with boundary on $S$ or a countinuous map $f$ from a closed Riemann surface into $M$. For an admissible parametrized surface $f$, let $[f]$ denote the homotopy class of $f$, where boundary is confined in $S$ along homotopies in the case that $f$ is a parametrized surface with boundary on $S$. Consider $C^{1}$ immersed admissible parametrized surfaces $f_{1}$ and $f_{2}$ such that $f_{1}$ and $f_{2}$ are nowhere tangent to $\partial M$ and they intersect at isolated points. Moreover, if $\left(f_{1}, z_{1}\right)$ and $\left(f_{2}, z_{2}\right)$ intersect at $p \in S$ for boundary points $z_{1}$ and $z_{2}$, then either they intersect transversally or are tangent to each other at $p$. We shall say that $f_{1}$ and $f_{2}$ intersect simply. Note that, given homotopy classes $\left\langle f_{1}\right\rangle$ and $\left\langle f_{2}\right\rangle$, there always exist $f_{1} \in\left\langle f_{1}\right\rangle$ and $f_{2} \in\left\langle f_{2}\right\rangle$ with the above properties. Indeed, we can actually choose $f_{1}$ and $f_{2}$ such that they intersect transversally.

Let $f_{1}$ and $f_{2}$ intersect simply. The intersection number (with signed mutiplicity) at an interior intersection point of $f_{1}$ and $f_{2}$ or at a transversal boundary intersection point is well-defined. For a nontransversal boundary intersection point $p$ of $\left(f_{1}, z_{1}\right)$ and $\left(f_{2}, z_{2}\right)$, we extend $f_{1}$ (near $\left.z_{1}\right)$ and $f_{2}$ (near $z_{2}$ ) across $S$ in the same fashion as in the proof of Lemma 5.2. The intersection number is then defined to be that of the extended maps.

Definition 7.1. Let $m_{1}$ denote the total number of boundary intersections of $f_{1}$ and $f_{2}$, and $m_{0}$ the total number of interior intersections. The intersection number $f_{1} \bullet f_{2}$ is defined to be $2 m_{0}+m_{1}$.

Lemma 7.2. $f_{1} \bullet f_{2}$ is a homotopy invariant.

Proof. Consider another immersion $\bar{f}_{1} \in\left\langle f_{1}\right\rangle$ which intersects $f_{2}$ simply. Choose a homotopy $f^{t}, 0 \leq t \leq 1$, between $f_{1}$ and $\bar{f}_{1}\left(f^{0}=f_{1}\right)$, which induces a map $F: \Sigma_{1} \times[0,1] \rightarrow M$. By suitable perturbations we can achieve the following: 1) $F$ is a $C^{1}$ immersion, 2) for each $t, f^{t}$ is nowhere tangent to $\left.\partial M, 3\right) f_{2}$ intersects $f^{t}$ transversally for each $t$ except finitely many parameters $t_{1}, \ldots, t_{m}$, and 4$)$ each $f^{t_{i}}$ intersects $f_{2}$ simply. (One can perturb $F$ so that $f_{2}$ intersects $F$ transversally, but that is not useful.) It is easy to see that $f^{t} \bullet f_{2}=f^{t^{\prime}} \bullet f_{2}$ for $t, t^{\prime} \in[0,1] \backslash\left\{t_{1}, \ldots, t_{m}\right\}$, provided that no $t_{i}$ lies between $t$ and $t^{\prime}$. Hence it suffices to show that $f^{t} \bullet f_{2}=$ $f^{t_{i}} \bullet f_{2}$ for $t$ near $t_{i}$. We can take $i=1$. Consider nontransversal intersection of $\left(f^{t_{1}}, z_{1}\right)$ and $\left(f_{2}, z_{2}\right)$ for $z_{1} \in \partial \Sigma_{1}$ and $z_{2} \in \partial \Sigma_{2}$. By the extension argument in the proof of Lemma 5.2, the intersection number $2 m_{0}+m_{1}$ counted in small neighborhoods of $z_{1}$ and $z_{2}$ is invariant under small deformation. On the other hand, transversal boundary intersections are obviously invariant. Next consider interior intersections of $f^{t_{1}}$ and $f_{2}$, whose total multiplicity we denote by $m_{0}$. For $t$ near $t_{1}$, they yield the interior intersections of $f^{t}$ and $f_{2}$ which have uniform positive distance (independent of $t$ ) from the boundary. The total multiplicity of the latter is clearly $m_{0}$. The desired equation follows.

It follows that $\left\langle f_{1}\right\rangle \bullet\left\langle f_{2}\right\rangle$ is well-defined. The results in [M1] and [M5] and the proof of Lemma 5.2 imply the following: 
Theorem 7.3 (Positivity of Intersections). Let $f_{1}$ and $f_{2}$ be $J$-holomorphic curves which are either closed or have boundary on $S^{*}$, and are nowhere tangent to $\partial M$. If they have distinct images and intersect each other, then $f_{1} \bullet f_{2}>0$.

Next we extend $\bullet$ to $J$-cusp-curves.

Definition 7.4. Let $f$ be a $J$-cusp-curve with boundary on $S^{*}$ and $f_{1}, \ldots, f_{m}$ its component $J$-holomorphic curves. We set $f \bullet f=\sum_{1 \leq i, j \leq m} f_{i} \bullet f_{j}$.

Gromov's compactness theorem (see [Y1] and Section 6) and a simple argument using homology in the interior lead to the following lemma.

Lemma 7.5. Let $f_{k}^{i}, i=1,2$, be two sequences of $J$-holomorphic curves such that $f_{k}^{i}, i=1,2$, have boundary on $S_{k}^{*}$ for a sequence of surfaces $S_{k} \subset \partial M$ which converges to a surface $S$ in $C^{1}$. Assume that $f_{k}^{i}$ converges to a $J$-cusp-curve $f^{i}$ with boundary on $S^{*}, i=1,2$, such that the boundary values of $f_{k}^{i}$ converge smoothly after being composed with suitable reparametrizations, i.e. no genuine boundary cusp arises. Then $f_{k}^{1} \bullet f_{k}^{2}=f^{1} \bullet f^{2}$ for large $k$.

This lemma holds true without assuming that there is no genuine boundary cusp.

For a Riemann surface $\Sigma$ with boundary, let $\mu(\Sigma)$ denote the Maslov class of the bundle pair $(T \Sigma, T \partial \Sigma)$. It's easy to see that

$$
\mu(\Sigma)=4-4 \gamma-2 l,
$$

where $\gamma$ denotes the genus of $\Sigma$ and $l$ the number of boundary compononts of $\Sigma$. Consider next a surface $S \subset \partial M$. We have

Theorem 7.6 (the Adjunction Formula). Let $f: \Sigma \rightarrow M$ be a J-holomorphic curve such that $f$ is an immersion along the boundary, $f(\stackrel{\circ}{\Sigma}) \subset \stackrel{\circ}{M}, f(\partial \Sigma) \subset S^{*}$, and $d f\left(T_{z} \Sigma\right) \not \subset T_{f(z)} \partial M$ for all $z \in \partial \Sigma$. Moreover, assume that $f$ does not factor through branched coverings of $\Sigma$, i.e. $f$ is not a multiple covering. Then the "embedding defect"

$$
D(f)=f \bullet f-\mu(f)+\mu(\Sigma)
$$

is a nonnegative even integer, and equals 0 if and only if $f$ is an embedding. Moreover, $D(f)$ is a multiple of 4 if $f$ is embedded along the boundary.

Remark. The general case that $f$ may have boundary branch points will be treated in a subsequent paper.

Proof. First assume that $f$ is an immersion. Then $\mu(f)=\mu_{o}(f)+\mu(\Sigma)$, where $\mu_{o}(f)$ is the normal Maslov class of $[f]$; see Definition 2.4. Consider the normal bundle $V$ of $f$ and the boundary bundle $V_{0}$ as in Section 2 (now $V$ is over $\Sigma$ and $V_{0}$ is over $\partial \Sigma$, as opposed to the embedding situation). Choose a $C^{1}$ section $\varphi$ of $V$ with boundary values in $V_{0}$ such that it has no zero along the boundary and intersects the zero section transversally. Moreover, we arrange that the zeros of $\varphi$ are all single points of $f$. (A single point of $f$ is a point $z \in \Sigma$ such that $f^{-1}((f(z))=z$. By the no factorization assumption and the asymptotical analysis in [Y1], there are only finitely many points which are not single points.) Then the argument in the proof of Proposition 2.7 implies that

$$
\mu_{o}([f])=2 m_{0},
$$

where $m_{0}$ is the number of signed zeros of $\varphi$. Now we consider $f_{1}=\exp (t \varphi)$ for a small $t$. The intersection number $f \bullet f$ is then given by counting the intersections 
of $f$ with $f_{1}$ (with weights). The total intersections resulting from the zeros of $\varphi$ are given by $2 m_{0}=\mu_{o}([f])$. Each interior double pair of $f$ gives rise to 2 interior intersection points between $f$ and $f_{1}$ with the same multiplicity, and each boundary double pair gives rise to 2 intersection points with the same multiplicity. (A double pair of $f$ is a pair of distinct points $\left(z_{1}, z_{2}\right)$ such that $f\left(z_{1}\right)=f\left(z_{2}\right)$.) By the results in [M1] and [M5], the multiplicity is positive in the former case. By the arguments in the proof of Lemma 5.2, the multiplicity in the latter case is also positive. Since $\mu(f)=\mu_{o}([f])+\mu(\Sigma)$, the desired conclusion follows.

Next consider the case that $f$ has branch points in the interior. Again by the asymptotic analysis in [Y1], $f$ has no self-overlapping. By the perturbation and patching arguments in [M1] and [M5], we can approximate $f$ by an immersed curve $f^{\prime}$, that coincides with $f$ near the boundary and is $J^{\prime}$-holomorphic for an almost complex structure which is $C^{1}$ close to $J$ (in particular it is homotopic to $J$ ) and coincides with $J$ near $\partial M$. Moreover, $f^{\prime}$ is not embedded. Since $f^{\prime} \bullet f^{\prime}=f \bullet f$ and $\mu\left(f^{\prime}\right)=\mu(f)\left(\mu\left(f^{\prime}\right)\right.$ is defined in terms of $\left.J^{\prime}\right)$, we arrive at the desired conclusion by applying the previous argument to $f^{\prime}$.

Remark. The above adjunction formula also covers closed $J$-holomorphic curves. Note that $D(f)$ is a multiple of 4 for closed $f$.

\section{Filling in the Presence of $J$-holomorphic Spheres}

In this section we prove Theorems 4,6 and 7 .

Proposition 8.1 (Smooth Compactness). Assume that $(M, J)$ has bounded geometry, $J$ is rationally regular, $\partial M$ is $J$-convex and $S \subset \partial M$ is a compact and orientable surface with finitely many complex points. Moreover, assume that no exceptional $J$-cusp-curve for $S$ exists. Let $\tilde{f}_{k}$ be a sequence of embedded (parametrized) $J$ holomorphic disks in $M$ with boundary on $S$ such that they have uniformly bounded area, their boundaries have uniformaly positive distance from the set of complex points of $S$, and the Maslov classes $\mu\left(f_{k}\right)=2$. In addition, we assume that for each $k$, the homology class $\left[\left.f_{k}\right|_{\partial B}\right]$ in $S$ is trivial. Then a subsequence of $\tilde{f}_{k} \circ \varphi_{k}$ converges smoothly to an embedded $J$-holomorphic disk $f$ with boundary on $S$, where $\varphi_{k}$ is a suitable sequence of conformal automorphisms of the unit disk.

Proof. We follow the proof of Proposition 6.1. Consider the normalized sequence $f_{k}$. The proof of Proposition 6.1 implies that the singular set $\Gamma$ is contained in the interior of $B$. Assume that $\Gamma$ is non-empty. Then the $J$-cusp-curve limit $f$ of $f_{k}$ consists of the $J$-holomorphic disk $f_{\infty}^{0}$ and non-constant $J$-holomorphic spheres $f_{\infty}^{1}, \ldots, f_{\infty}^{m}$ for some $m \geq 1$. The cited proof also implies that $f_{\infty}^{0}$ is an embedding along the boundary. By Gromov's compactness theorem and the assumption on the Maslov classes we have $\mu(f)=2$. By Theorem 7.6 and Lemma 7.5,

$$
f \bullet f-\mu(f)+2=0 .
$$

To proceed, we introduce some terminology. A simple $J$-holomorphic sphere $f^{(1)}$ is called a generator of a $J$-holomorphic sphere $f^{(2)}$, if $f^{(2)}$ equals the composition of $f^{(1)}$ with a surjective holomorphic self-map $\varphi$ of $\mathbb{S}^{2}$. The degree of $\varphi$ is called the degree of $f^{(2)}$. Let $\mathcal{F}=\left\{f^{1}, \ldots, f^{s}\right\}$ be a maximal collection of distinct generators of the component spheres of $f$. The multiplicity $k_{i}$ of $f^{i}$ is defined to be the sum of the degrees of those spheres in $f$ which are generated by $f^{i}$. We set $f^{0}=f_{\infty}^{0}$. 
By Theorem 7.3, we have, for each $i$ and all $k, f^{i} \bullet f_{k} \geq 0$, whence $f^{i} \bullet f \geq 0$. But $f \bullet f=0$, which follows from (8.1). We deduce that $f^{i} \bullet f=0$ for each $i$. Then the connectedness of image $(f)$ and positivity of intersections (Theorem 7.3) imply that each $f^{i}$ has negative self-intersection number. Applying the rational regularity assumption, Lemma 3.7, and the adjunction formula, we conclude that each $f^{i}, i \geq 1$ is an exceptional $J$-holomorphic sphere; in particular, $f^{i} \bullet f^{i}=-2$ and $\mu\left(f^{i}\right)=2$. Consequently, the identities $f^{i} \bullet f=0$ imply

$$
\begin{aligned}
& f^{1} \bullet f^{0}+k_{2} f^{1} \bullet f^{2}+\ldots+k_{s} f^{1} \bullet f^{s}=2 k_{1}, \\
& f^{2} \bullet f^{0}+k_{1} f^{2} \bullet f^{1}+\ldots+k_{s} f^{2} \bullet f^{s}=2 k_{2}, \\
& \ldots \ldots, \\
& f^{s} \bullet f^{0}+k_{1} f^{s} \bullet f^{1}+\ldots+k_{s-1} f^{s} \bullet f^{s-1}=2 k_{s} .
\end{aligned}
$$

We claim that $f^{i} \bullet f^{0}>0$ and hence $f^{i} \bullet f^{0} \geq 2$ for each $i \geq 1$. Indeed, assume e.g. $f^{1} \bullet f^{0}=0$. Then $f^{1}$ must intersect one of the other spheres, say $f^{2}$, because image $(f)$ is connected. Then $f^{1} \bullet f^{2} \geq 2$, and hence (8.2) along with positivity of intersections implies $f^{1} \bullet f^{0}=f^{1} \bullet f^{3}=\ldots=f^{1} \bullet f^{s}=0$ and $f^{2} \bullet f^{0}=f^{2} \bullet f^{3}=\ldots=f^{2} \bullet f^{s}=0$. This implies that $f^{1}$ and $f^{2}$ are disjoint from the remaining curves in $f$, contradicting the connectedness of image $(f)$. Now, since $f^{i} \bullet f=0$ and $f^{i} \bullet f^{i}=-2$ for $i \geq 1$, we deduce from (8.1) that

$$
\left(2+f^{0} \bullet f^{0}-\mu\left(f^{0}\right)\right)+\sum_{i=1}^{s}\left(f^{0} \bullet f^{i}-2\right)=0 .
$$

By the above reasoning, the sum on the left hand side of (8.3) is nonnegative. By Theorem 7.6, the bracket on the left hand side of (8.3) is also nonnegative. Hence they both vanish. It follows that $f^{0}$ is an embedding and $f^{i} \bullet f^{0}=2$ for each $i \geq 1$. Applying (8.2) once again we then derive that $f^{i} \bullet f^{j}=0$ for all distinct $i, j \geq 1$ and $k_{i}=1$ for all $i$. We conclude that $f$ is an exceptional $J$-cusp-curve for $S$. This is impossible by the assumptions. Hence the singular set $\Gamma$ is empty. Consequently, $f_{k}$ converges smoothly to $f_{\infty}^{0}$. The proof of Proposition 6.1 then implies that $f_{\infty}^{0}$ is an embedding.

Proof of Theorem 6. By Lemma 3.7 and Proposition 3.8, if $J$ is rationally $S$-regular in the embedding set-up or parametric set-up, then $\mu\left(f^{0}\right) \geq 1$ for an arbitary embedded (paramertrized) $J$-holomorphic disk $f^{0}$ with boundary on $S^{*}$. Hence no exceptional $J$-cusp-curve for $S$ exists, and the result follows from Theorem 4 . (In the case of the parametric set-up, it is not necessary to use Theorem 4, because no nontrivial $J$-cusp-curve with Maslov class 2 exists. Then the result follows from the proof of Theorem 2 and Gromov's compactness theorem (convergence of the Maslov classes).)

Proof of Theorem 4. The first statement follows immediately from Proposition 8.1 and the proof of Theorem 2. Next we prove a result which is stronger than the second statement. Assume that there is an exceptional $J$-cusp-curve $C$ for $S$. We claim that there exists no smooth 1-parameter family of $J$-holomorphic disks $f_{t}, 0<$ $t<1$, with boundary on $S$ such that $f_{t}(\partial B)$ converges to a point $p_{1} \in S \backslash \bigcup_{t} f_{t}(\partial B)$ as $t \rightarrow 0$ and a point $p_{2} \in S \backslash \bigcup_{t} f_{t}(\partial B)$ as $t \rightarrow 1$, and that $\left(\bigcup_{t} f_{t}(\partial B)\right) \cup\left\{p_{1}, p_{2}\right\}=$ $S$. Assume that such a family exists. Then $p_{1}$ and $p_{2}$ are necessarily the two complex points of $S$, as can easily be shown by using Lemma 5.4 and Lemma 5.5. Proposition 5.6 then implies that $f_{t}$ is given by Theorem 1 for $t$ near 0 or 1 . Consequently, all $f_{t}$ have Maslov class 2. Moreover, the proof of Theorem 2 implies 
that all $f_{t}$ are embeddings, whence $f_{t} \bullet f_{t}=0$ by Theorem 7.6. On the other hand, a global uniqueness similar to Proposition 6.2 holds, which implies that the disk $f$ in $C$ is a covering of $f_{t}$ for some $t$. We deduce that $f \bullet f=0$, a contradiction.

Now we proceed to prove Theorem 7 . Let $M, J$ and $S$ satisfy the conditions in Theorem 7.

Consider a $J$-cusp-curve $f$ whose component $J$-holomorphic curves are either disks or spheres. The concept of generators introduced in the proof of Proposition 8.1 naturally extends to disks. Let $f^{0}, f^{1}, \ldots, f^{s}$ be a maximal collection of distinct generators of $f$ with multiplicities $k_{i}$. Let $\pi\left(f^{i}\right)$ denote the equivalence class of $J$-holomorphic curves which are compositions of $f^{i}$ with automorphisms of $S^{2}$ or $B$. In the following definition $f^{i}$ stands for $\pi\left(f^{i}\right)$.

Definition 8.2. The formal sum $\sum_{0 \leq i \leq s} k_{i} f^{i}$ is called the "J-class" of $f$. The formal sum $\sum_{0 \leq i \leq s} f^{i}$ is called the "reduction" of $f$. We say that $f$ is "irreducible", if its $J$-class is the same as its reduction. A $J$-cusp-curve $f_{1}$ whose $J$-class is the same as the reduction of $f$ is called a "reduced $J$-cusp-curve of $f$ ". Two $J$-cuspcurves with the same $J$-class are said to be $J$-equivalent.

Lemma 8.3. Let $f$ be a J-cusp-curve which is either closed or has its boundary on $S^{*}$. Assume that $f$ is the limit of a sequence of $J$-holomorphic curves $f_{k}$ (with boundary on $S^{*}$ in the latter case) such that image $(f) \neq i$ image $\left(f_{k^{\prime}}\right)$ for a subsequence $f_{k^{\prime}}$. Then $f \bullet f \geq 0$. Consequently, a sequence of J-holomorphic curves $f_{k}$ with negative self-intersection number cannot converge to a nontrivial J-cusp-curve unless image $\left(f_{k}\right)$ are all identical and $f_{k}$ are multiple coverings for large $k$.

Proof. Let $f^{\prime}$ be an arbitary component $J$-holomorphic curve in $f$. Theorem 7.3 implies that $f^{\prime} \bullet f_{k} \geq 0$ for all $k$, whence $f^{\prime} \bullet f \geq 0$. Then $f \bullet f \geq 0$.

Definition 8.4. A $J$-holomorphic disk $f_{0}$ with boundary on $S$ is called "balanced", provided that either $f_{0} \bullet f_{0}=0$, or there is a $J$-cusp-curve $f$ consisting of $f_{0}$ and several (at least one) $J$-holomorphic spheres $f_{1}, \ldots, f_{k}$ such that $f \bullet f=0$ and $f_{i} \bullet f \geq 0,0 \leq i \leq k$ (it follows that $f_{i} \bullet f_{i}<0$ for all $i$ ). In the latter case, $f$ is called a "balance" of $f_{0}$ and we say that $f_{0}$ is nonstandard.

Definition 8.5. A "singular filling" of $S$ is a one-parameter family $\mathcal{F}$ of $J$-holomorphic disks $f_{t}, 0<t<1$, with boundary on $S^{*}$ such that the following hold:

1) for $t$ near 0 and $1, f_{t}$ are given by the local filling families near the two complex points $p_{1}$ and $p_{2}$ of $S$ provided by Theorem 1;

2) the $f_{t}$ are mutually disjoint;

3) there is a finite set $\mathcal{T}=\left\{t_{1}, \ldots, t_{m}\right\} \subset(0,1)$ such that $f_{t}$ is an embedding for all $t \notin \mathcal{T}$, and $\left\{f_{t}, t \notin \mathcal{T}\right\}$ yields a smooth embedding of $B \times(0,1) \backslash \mathcal{T}$;

4) all $f_{t}$ are embeddings along the boundary, and $\left.f_{t}\right|_{\partial B}, 0<t<1$, yield a smooth family of embedded curves in $S$ such that $\bigcup_{t}$ image $\left(\left.f_{t}\right|_{\partial B}\right)=S \backslash\left\{p_{1}, p_{2}\right\}$;

5) all $f_{t}$ are balanced;

6) the $f_{t}, t \notin \mathcal{T}$, are mutually homotopic with boundary confined in $S^{*}$.

(It follows that $f_{t} \bullet f_{t}=0$ for $t \notin \mathcal{T}$ and $f_{t} \bullet f_{t}<0$ for $t \in \mathcal{T}$.)

The $t_{i} \in \mathcal{T}$ are called "singular parameters" and $f_{t_{i}}$ "singular leaves". A reparametrization of $\mathcal{F}$ is a collection of orientation-preserving diffeomorphisms $h_{i}:\left(t_{i-1}, t_{i}\right) \rightarrow\left(t_{i-1}, t_{i}\right), i=1, \ldots, m+1$, with $t_{0}=0, t_{m+1}=1$.

Proof of Theorem \%. Since both $S$ and $\partial M$ are orientable, we can find a sequence of simple surfaces $S_{k} \subset \partial M$ which are disjoint from $S$ and converge smoothly to 
$S$. By Theorem 6, modulo small perturbations we can assume that the conclusion of Theorem 2 holds for all $S_{k}$. Choose integral curves $\mathcal{L}_{i}^{k}, i=0,1,2$, of the characteristic direction on $S_{k}^{*}$ and the associated parametrizations $\beta_{i}^{k}, i=0,1,2$, as in the proof of Theorem 2, such that they converge to corresponding $\mathcal{L}_{i}$ and $\beta_{i}$ on $S$ respectly. For each $k$, let $f_{t}^{k}, 0<t<1$, be the filling family for $S_{k}$ as constructed in the proof of Theorem 2 . Recall that the $f_{t}^{k}$ satisfy a 3 -point condition: $f_{t}^{k}\left(z_{i}\right)=\beta_{i}^{k}(t), i=0,1,2$.

Fix $t$ and set $f^{k}=f_{t}^{k}$. By Gromov's compactness theorem (with varing supporting totally real submanifolds, see [Y1]), there is a subsequence, still denoted $f^{k}$, which converges to a $J$-cusp-curve $f$. The proof of Proposition 6.1 implies that $f$ consists of a disk $f_{0}$ with embedded boundary and (possibly) several spheres. By the 3-point condition, $f^{k}$ converges to $f_{0}$ smoothly away from a set of finitely many interior points, which is empty provided that $f$ consists of $f_{0}$ only. In particular, $f_{0}$ satisfies the 3-point condition. By the properties of $S_{k}$, the intersections of $f_{0}$ and $f^{k}$ can only occur in the interior. We define the intersection numbers $f_{0} \bullet f^{k}=\left\langle f_{0}\right\rangle \bullet\left\langle f^{k}\right\rangle$ in a similar way to Section 7. By the results in [M1] we have $f_{0} \bullet f^{k} \geq 0$.

We claim that $f_{0} \bullet f \geq 0$. To see this, we choose an immersed surface $f_{0}^{\star}$ with boundary on $S^{*}$ which is homotopic to $f_{0}$ with boundary confined in $S^{*}$ and whose boundary is disjoint from that of $f_{0}$. Then the intersection number $f_{0} \bullet f^{k}$ can be computed in the following way. We embed $M$ into a suitable manifold $M_{\infty}$ such that there are closed surfaces $\tilde{f}_{0}, \tilde{f}_{0}^{\star}$ and $\tilde{f}^{k}$ in $M_{\infty}$ which are obtained from $f_{0}$, $f_{0}^{\star}$ and $f^{k}$ respectively by gluing suitable disks $D_{0}, D_{0}^{\star}$ and $D^{k}$ to them along the boundary. Moreover, 1) $D_{0}^{\star}$ is disjoint from $D_{0}$ and all $D^{k}$, and 2) $D^{k}$ converges to $D_{0}$ smoothly as $k \rightarrow \infty$. It follows that for $k$ large, the homology class $\left[\tilde{f}^{k}\right]_{\infty}$ equals $[f]_{\infty} \equiv$ the sum of $\left[\tilde{f}_{0}\right]_{\infty}$ and the homology classes generated by the spheres in $f$, where [ $]_{\infty}$ denotes homology class in $M_{\infty}$. We have $f_{0} \bullet f^{k}=2\left[\tilde{f}_{0}^{\star}\right]_{\infty} \cdot\left[\tilde{f}^{k}\right]_{\infty}$, as can easily be verified. Moreover, $f_{0} \bullet f=2\left[\tilde{f}_{0}^{\star}\right]_{\infty} \cdot[f]_{\infty}$. It follows that $f_{0} \bullet f^{k}=f_{0} \bullet f$ for large $k$, whence $f_{0} \bullet f \geq 0$. Similarly, $h \bullet f \geq 0$ for every sphere $h$ in $f$. Since $f \bullet f=0$, which follows from Theorem 7.6 and Lemma 7.5, we deduce as in the proof of Proposition 8.1 that all component curves in $f$ have negative selfintersection number. It follows that $f$ is a balance of $f_{0}$.

Claim 1. The limit $f_{0}$ is independent of the (sub)sequence $f^{k}$.

To prove it, we consider another such limit $f_{0}^{\prime}$ and its balance $f^{\prime}$ which arise from another sequence (or subsequence) $f^{k, 1}$. For each $k$, the intersection of $f^{k}$ with $f_{0}^{\prime}$ is nonnegative, and so is its intersection with every possible sphere in $f^{\prime}$. On the other hand, it is not hard to see that $f^{\prime} \bullet f^{k}=0$. Hence the said intersections all vanish. It follows that the intersections of $f_{0}^{\prime}$ and all the possible spheres in $f^{\prime}$ with $f$ are zero. If $f_{0}^{\prime}$ intersects at least one sphere in $f$ positively, then $f_{0}^{\prime} \bullet f_{0}$ is negative. By Theorem 7.3, these two disks have the same image. By the 3-point condition, they are identical. If $f_{0}^{\prime}$ is disjoint from all the spheres in $f$ (or there is no sphere in $f$ ), then $f_{0}^{\prime} \bullet f_{0}=0$. Since these two disks intersect each other by the 3-point condition, we again deduce that they have the same image. Then they coincide with each other. The claim follows. Note that if $f$ contains no sphere, neither does $f^{\prime}$ (and vice versa). To indicate the dependence on $t$, we set $f_{t}=f_{0}$.

We shall say that a parameter $t$ is singular, provided that the above $f$ contains spheres, i.e. $f_{0}$ is nonstandard. Let $\mathcal{T}$ denote the set of singular parameters. Recall that $f_{t} \bullet f_{t}<0$ for $t \in \mathcal{T}$. 
Claim 2. The $f_{t}$ are mutually disjoint.

Indeed, we can apply the above argument for Claim 1 to any given pair $f_{t}, f_{t^{\prime}}$ with $t^{\prime} \neq t$ to show that $f_{t} \bullet f_{t^{\prime}} \leq 0$. But these two disks have distinct images, and so they must be disjoint.

Claim 3. $\mathcal{T}$ is finite.

Assume that there are infinitely many singular parameters. They cannot accumulate at 0 or 1 . Indeed, by the uniqueness part of Theorem 2 and Theorem 6 , for each $k$, the filling disks for $S_{k}$ near its two elliptic complex points are given by the construction in the proof of Theorem 1. This construction provides a smooth control of these disks. Since the $S_{k}$ converge smoothly to $S$, this smooth control is independent of $k$. It follows that these disks converge smoothly as $k$ goes to infinity. Hence there is no singular parameter near 0 or 1 . Now choose a sequence of distinct singular parameters $t_{i}$ which converges to some $t \in(0,1)$. By Gromov's compactness theorem and Lemma 8.3, by passing to a subsequence we can assume that $f_{t_{i}}$ converges smoothly to some $f_{\infty}$. Arguing as before we deduce that $f_{\infty}=f_{t}$. Hence $f_{t_{i}} \bullet f_{t}=f_{t} \bullet f_{t}<0$ for large $i$. This contradicts Claim 2 .

It is easy to see that the $f_{t}, t \notin \mathcal{T}$, are mutually homotopic with boundary confined in $S^{*}$. Using the above arguments, it is also easy to show that for each $t_{0} \in(0,1), f_{t}$ converge smoothly to $f_{t_{0}}$ in a neighborhood of $\partial B$. Finally, the proof of Theorem 2 can be applied to show that $f_{t}, t \notin \mathcal{T}$, yields a smooth embedding of $B \times(0,1) \backslash \mathcal{T}$. We conclude that $\mathcal{F}=\left\{f_{t}, 0<t<1\right\}$ is a singular filling of $S$.

The uniqueness follows from Theorem 8.8, below.

If $\mathcal{F}$ has a singular leaf $f_{t}$, then the proof of Proposition 8.1 implies that its balance $f$ as obtained above is an exceptional $J$-cusp-curve for $S$. On the other hand, if exceptional $J$-cusp-curves exist, then the uniqueness implies that their disks must occur as singular leaves of $\mathcal{F}$.

The next results provide an analysis of the singularity structure of the singular filling.

Proposition 8.6. Let $\mathcal{F}$ be the singular filling for $S$ and $t_{0} \in \mathcal{T}$ a singular parameter. Let $t_{k}$ be a sequence converging to $t_{0}$. Then a subsequence of $f_{t_{k}}$ converges to a balance $f$ of $f_{t_{0}}$. It also converges to $f_{t}$ smoothly away from finitely many interior points.

The proof is quite straightforward; we omit it. Note that $f$ can be understood as a blow-up limit of the map $\Phi$ at the singular parameter $t_{0}$, where $\Phi(z, t)=$ $f_{t}(z), t \in(0,1) \backslash \mathcal{T}$. The $J$-class of $f$ is called a $J$-blow-up of $\mathcal{F}$ at $t$.

Theorem 8.7 (Unique Blow-up). At each singular parameter, there is a unique $J$-blow-up. Moreover, the associated $J$-cusp-curve is an exceptional $J$-cusp-curve for $S$.

Proof. Consider two blow up limits $f$ and $f^{\prime}$ at a singular parameter $t$. The arguments in the proof of Theorem 7 imply that $f^{i} \bullet f^{\prime}=0$ for any generator $f^{i}$ of $f$. It follows that $f^{i}$ must also be a generator of $f^{\prime}$. Similarly, every generator of $f^{\prime}$ is a generator of $f$. Hence the reduction of $f$ is the same as that of $f^{\prime}$. On the other hand, the proof of Proposition 8.1 implies that $f$ is an exceptional $J$-cusp-curve.

Finally we have the following 
Theorem 8.8 (Global Uniqueness). Let $f$ be a nonconstant $J$-holomorphic curve with nonempty boundary on $S$. Assume that $\left\{p_{1}, p_{2}\right\} \not \subset$ image $(f)$, where $p_{1}$ and $p_{2}$ are the two complex points of $S$. Then $f$ is the composition of some $f_{t}$ with an automorphism of $B$ or a branched covering of $B$.

Proof. $f$ must intersect some $f_{t_{0}}$. If $\operatorname{image}(f)=i$ mage $\left(f_{t_{0}}\right)$, then we are done. Otherwise, $f \bullet \tilde{t}_{t_{0}}>0$, where $\tilde{f}_{t_{0}}=f_{t_{0}}$ if $t_{0} \notin \mathcal{T}$, and it is a $J$-cusp-curve associated with the $J$-blow-up at $t_{0}$ otherwise. Since at least one of $p_{1}$ and $p_{2}$ is not contained in image $(f), f$ is disjoint from $f_{t_{1}}$ for (a nonsingular parameter) $t_{1}$ near 0 or 1 . Choose such a $t_{1}$. We have $f \bullet f_{t_{1}}=0$. But the structure of singular filling, Theorem 8.7 and Lemma 7.5 imply that $f \bullet f_{t_{1}}=f \bullet f_{t_{0}}$, a contradiction.

\section{REFERENCES}

[A] M. Audin, Symplectic and almost complex manifolds, in Holomorphic Curves in Symlectic Geometry, by M. Audin and J. Lafontaine (editors), Birkhäuser, 1994, pp. 41-74. MR 95i: 58005

[AA] F. J. Almgren and W. K. Allard, On the radial behavior of minimal surfaces and the uniqueness of their tangent cones, Ann. Math. 113 (1981), 215-265. MR 83k:49069

[B] E. Bishop, Differentiable manifolds in complex Euclidean space, Duke Math. J. 32 (1965), 1-22. MR 34:369

[BG] E. Bedford and B. Gaveau, Envelopes of holomorphy of certain 2-spheres in $\mathbb{C}^{2}$, Amer. J. Math. 105 (1983), 975-1009. MR 84k:32016

[BK] E. Bedford and W. Klingenberg, On the envelope of holomorphy of a 2-sphere in $\mathbb{C}^{2}$, J. Amer. Math. Soc. 4 (1991), 623-646. MR 92j:32034

[E1] Y. Eliashberg, Filling by holomorphic disks and its applications, Geometry of Lowdimensional Manifolds, 2 (Durham, 1989), London Math. Soc. Lecture Note Ser., vol. 151, Cambridge Univ. Press, 1990, pp. 45-67. MR 93g:53060

[E2] Y. Eliashberg, Topology of 2-knots in $\mathbb{R}^{4}$ and symplectic geometry, The Floer Memorial Volume, Progr. Math., vol. 133, Birkhäuser, Basel, 1995, pp. 335-353. MR 96j:57023

[F] A. Floer, The unregularized gradient flow of the symplectic action, Comm. Pure Appl. Math. 41 (1988), 775-813. MR 89g:58065

[G] M. Gromov, Pseudo-holomorphic curves in symplectic manifolds, Invent. Math. 82 (1985), 307-347. MR 87j:53053

$[\mathrm{H}] \quad$ H. Hofer, Pseudo holomorphic spheres in symplectizations with applications to the Weinstein conjecture in dimension three, Invent. Math. 114 (1993), 515-563. MR 94j:58064

[M1] D. McDuff, The local behavior of holomorphic curves in almost complex 4-manifolds, J. Diff. Geom. 34 (1981), 143-164. MR 93e:53050

[M2] D. McDuff, Singularities of J-holomorphic curves in almost complex 4-manifolds, J. Geom. Anal. 2 (1992), 249-266. MR 93g:58032

[M3] D. McDuff, The structure of rational and ruled symplectic 4-manifolds, J. Amer. Math. Soc. 3 (1990), 679-712; erratum, 5 (1992), 987-988. MR 91k:58042; MR 93k:58098

[M4] D. McDuff, Examples of symplectic structures, Invent. Math. 89 (1987), 13-36. MR 88m:58061

[M5] D. McDuff, Singularities and positivity of intersections of J-holomorphic curves in symplectic 4-manifolds, in Holomorphic Curves in Symplectic Geometry, edited by M. Audin and J. Lafontaine (editors), Birkhäuser, 1994, pp. 191-215. MR 95i:58005

[MS] D. McDuff and D. Salamon, Symplectic Geometry, Lecture Notes, Cambridge, 1993.

[MT] D. McDuff and L. Traynor, Cambridge Univ. Press, 1993, pp. 169-182 in Symplectic Geometry. MR 95j:58052

[MW] M. J. Micallef and B. White, The structure of branch points in area minimizing surfaces and in pseudoholomorphic curves, preprint. Ann. Math. 141 (1995), 35-85. MR 96a:58063

[SU] J. Sacks and K. Uhlenbeck, The existence of minimal immersions of 2-spheres, Ann. Math. (2) 113 (1981), 1-24. MR 82f:58035

[S1] L. Simon, Asymptotics for a class of non-linear evolution equations, with applications to geometric problems, Ann. Math. (2) 118 (1983), 525-572. MR 85b:58121 
[S2] L. Simon, Cylindrical tangent cones and the singular set of minimal submanifolds, J. Diff. Geom. 38 (1993), 585-652. MR 95a:58026

[S3] L. Simon, On the singularities of harmonic maps, preprint.

[V] I. N. Vekua, Generalized analytic functions, Pergamon Press, Oxford, and Addison-Wesley, Reading, MA, 1962. MR 27:321

[Y1] R. Ye, Gromov's compactness theorem for pseudo holomorphic curves, Trans. Amer. Math. Soc. 342 (1994), 671-694. MR 94f:58030

[Y2] R. Ye, Filling by holomorphic disks in symplectic 4-manifolds, preprint, Centre de Mathmatiques et de Leurs Applications, ENS Cachan, 1994.

Department of Mathematics, University of California, Santa Barbara, California 93106

E-mail address: yer@math.ucsb.edu 